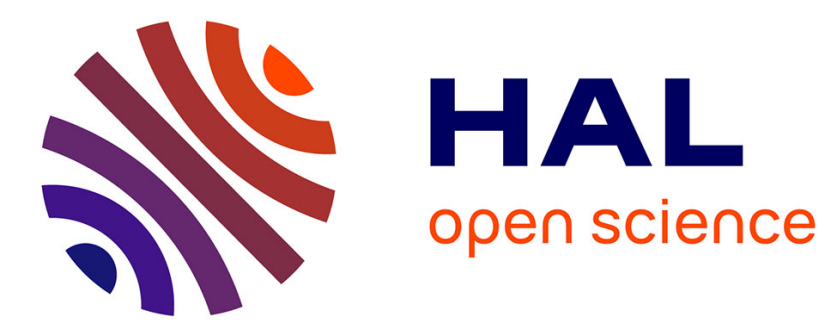

\title{
Using occluding contours for 3D object modeling
}

Régis Vaillant, Olivier Faugeras

\section{To cite this version:}

Régis Vaillant, Olivier Faugeras. Using occluding contours for 3D object modeling. [Research Report] RR-1325, INRIA. 1990. inria-00075235

\section{HAL Id: inria-00075235 https://hal.inria.fr/inria-00075235}

Submitted on 24 May 2006

HAL is a multi-disciplinary open access archive for the deposit and dissemination of scientific research documents, whether they are published or not. The documents may come from teaching and research institutions in France or abroad, or from public or private research centers.
L'archive ouverte pluridisciplinaire HAL, est destinée au dépôt et à la diffusion de documents scientifiques de niveau recherche, publiés ou non, émanant des établissements d'enseignement et de recherche français ou étrangers, des laboratoires publics ou privés. 


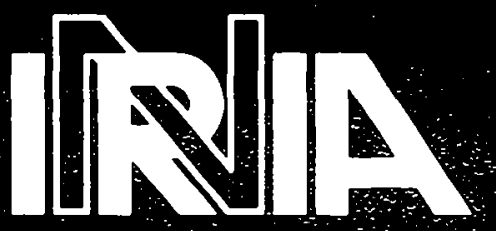

UNITÉ DE RECHERCHE NRIA-SOPHIA ANTIPOLIS

\section{Rapports de Recherche}

$N^{\circ} 1325$

Programme 6

Robotique, Image et Vision

USING OCCLUDING CONTOURS FOR 3D OBJECT MODELING

Régis VAILLANT

Olivier D. FAUGERAS

Domaine de Voluceau

Rocquencouit

$$
\text { B.P105 }
$$

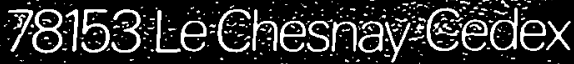

\section{France}

Tél:(1)39635511 



\title{
Les contours d'occultation: détection et re- construction en vue de la modélisation d'ob- jets $3 D^{1}$
}

\author{
Régis Vaillant - Olivier D. Faugeras \\ INRIA Sophia Antipolis \\ 2004 Route des Lucioles \\ 06565: Valbonne C'edex \\ regis İmirsa.inria.fr - fangerasị̂mirsa.in ria.fr
}

\begin{abstract}
Ce rapport de reclerche est consacré a l'ctude des contours d'occultation. Les contours d'occultation sont coux pour lescuels le rayou optiçue est tangent à la surface observie. Nous présentons plusieurs résultats liés aux propriétés de lïmage de ces contours et un algorithme permettant : la détection des contonrs dooccultation en utilisant plusieurs images (au moins 3) et la reconstruction le la surface observée dans le voisinage du contour. Ja reconstruction fajte comprend les proprićtés différenticlles de la surface juscula l'ordre 2: courbures el directions principales. Nous présentons aussi un algorithne tòs simple permettant le calcul du signe de la courbure gaussienne. Des résultats sur des domnécs synthétiques (en vue d’évaluer la qualité numérigue et des donnćes réelles sont préscntées. Les applications potentielles de ces algorit hmes sont très importantes dans le cadre des problèmes de modólisation dobjets.
\end{abstract}

\section{Using Occluding Contours for 3D Object Modeling}

This research report is derotol to the stuly of the extremal boundaries. The extremal boundaries are such that the optical ray is tangent to the surface. We present several results about the properties of the image of these contours and an algorithm for detecting and reconstructing them (we nced at least three images). The reconstruction includes up to second order differential properties (principal curvatures and directions). We also present an algorithm for computing very sinuply the sign of the gaussian curvature. We show results on synthetic (it is a very convenient way to study the mumerical quality of the results) and real data. Potemtial applications of this kind of algorithm are in the morleling of $3 \mathrm{I}$ ) objects.

\footnotetext{
${ }^{1}$ This work was supporled in part by Exprit projece p'2502. I biles
} 


\section{Introduction}

One of the aims of compule vision is to extract concise surface descriptims from several images of a scence. The descriptions can be used for the purpose of object recoguition and for geomertic reasoning (such as obstacle avoidance).

Stereovision is often used for recovering the structure of the 3D workl. Standard rechuigues can determine the depth of edges on a surface. These techniques fail with cxtremal bomularics as thesc change according to the viewpoint.

When we form on the retina of a camera an image of the environment we assume. 1up to a rery good approximation, that it is a perspective projection of the scene and that. at crery pixel, the image intensity is proportional to the scene irradiance [Hor86]. One of the goals of computer vision is to extract from this pattern of changing intensities relevant information about the three-dinensional geometry and kinematics of the objects present. One of the key ideas to achieve this is to extract edges because they always signal important physical properties of the scene: discontinuitics in the reflectance, texture, color, depth, motion are among the man! possibilities. But even though the knowledge of the physical origin of an edge in an image is extremely important for further analysis, this information is usually lost making llue task of computer vision more difficult.

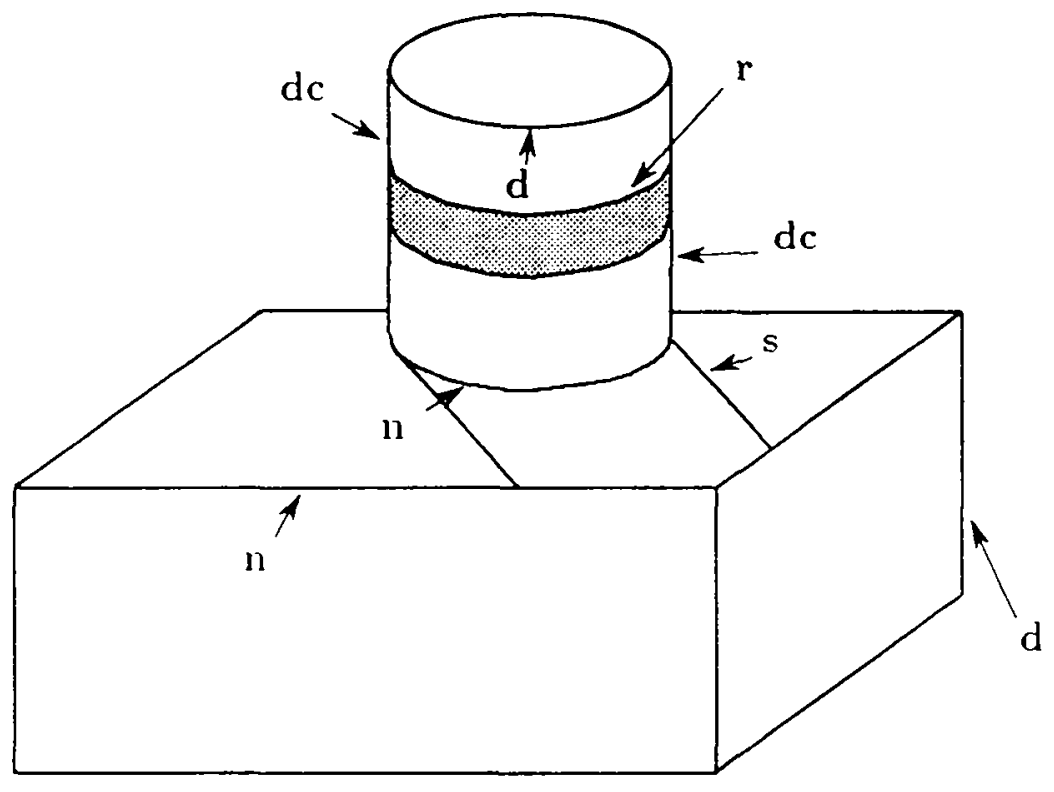

Figure 1: The different edges

For example. figure 1 shows examples of several of these edges: edges labeled $\mathbf{r}$ correspond to a rapid change of the reflectance of the cylinder, edges labeled s are caused by the shadow of the cylinder cast on the parallelepipedic box that supports it. edges labeled $\mathbf{d}$ correspond to discontinuitics of the distance of the object to the camera, the nomal to the object being discontinuous there (peaks and throughs), edges labeled $n$ signal a discontinuity of the normal to the object without a discontinuity of the distance. Finally edges labelled de signal a discontinuity of the distance to the camera with no discontinuity of the normal to the object which varies smoolly in the vicinty of the edge. 
Labelling correct!y and robustly those edges has proved to be a formidable task from just one image. If we have several images of the same scene taken from slightly different viewpoim as in the case of a moving observer or in the case of sterco, then the task may be a little simpler.

Let us consider for example the case of stereo. Many algorithms proposed for doing sterco are celge based [Ciri85.BB81,0K85,AL87],... After matching these edges they can provide depult along the edges which can be interpolated to yield a surface representation of the scene [Ciris:3. FLB90]. Classification of the edges can then be obtained from this surface model.

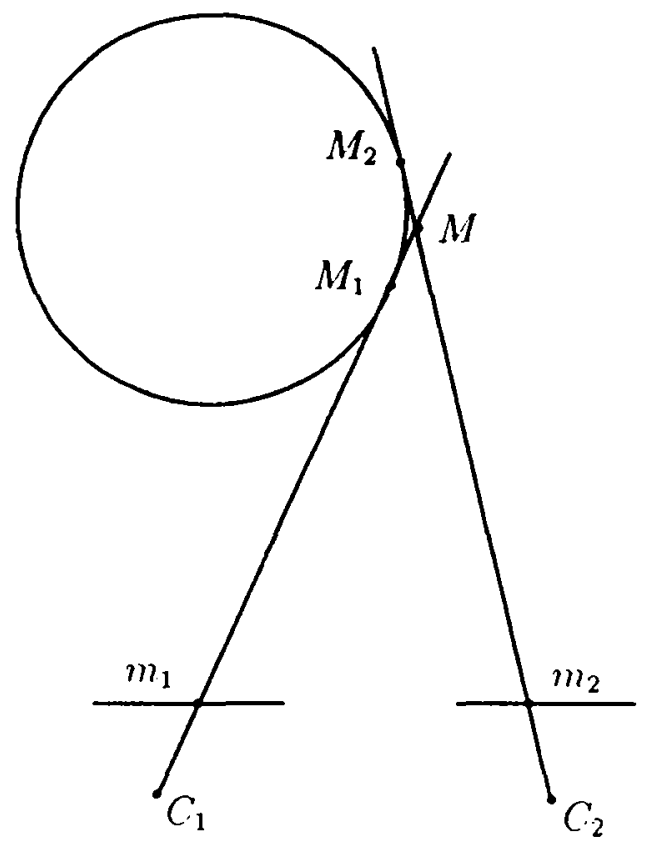

Figure 2: pixels $m_{1}$ and $m_{2}$ are not the images of the same physical point on the cylinder

This sounds like a reasonable thing 10 do but be cautious! This analysis assmunes that an edge in an image which is matched to an edge in another image both correspond to the images of the same physical event in the 3D scene. Unfort unately, this is not true of the edges labeled dc in figure 1 as the reader may convince himself by looking at figure 2 which represents the stereo geometry in a plane going through the optical centers of the two cameras. Pixels $m_{1}$ and $m_{2}$ are both on the outlinc of the cylinder as seen from the two cameras but because of the geometry of the object they are not the images of the same physical point: if our stereo algorithm matches them they will yield the reconstructed point $M$ which is an error.

Such edges have received several nanes in the literature: some authors call them obscuring edges, extremal boundaries when refering to their images, or the rim when referring to them in 3D. Note that the rim is not a physical marking on the object but depends both on its geometry and the viewing position.

It looks therefore as if we should pay attention to those edges and that further processing of the data is necessary in their vicinity. We may take the pessinistic view of abandoning the hope of obtaining an accurate reconstruction of the object near those edges. As we show in this paper, this would be a big blunder since it is precisely there that we can ext ract the most information about the geometry of the object: unlike edges such as $d, r$. s. or 11 where only the distance ds to the caneras can be recovered. extremal boundaries allow us to recover the 
distance, the normal $n \leq$ of the object and the principal curvatures or in terms of differential geometry, the differential properties of order 0,1 and 2 .

If this statement is correct (and we show that it is in the rest of the paper) obscuring edges a ppear more as a forgotten or lost treasure than as a nuisance for computer vision as a superficial analysis might conclude.

In this paper, we propose a new method for detecting extremal boundaries. We also proposs' an algorithm for reconstructing exactly the curves observed by each camera and computing the principal curvatures of the object surface in their vicinity.

Extremal boundaries have al ready been studied by several authors. They broadly fall into (wo classes; in the first class, the anthors assume that they have one monocular image in which they have been able to identify by means that they do no describe one occluding edge. They then go about to describe the kind of inferences that can be drawn from this observation. Marr [Mar\&2] already signaled their possible importance to infer the sign of the gaussian curvature of the object. His observations were made more precisely by Koenderink [Koe84] who has done a fairly thorough job at analyzing the qualitative properties of the rim and its images. Braly et. al [BPYAS5] have derived other ways some of proving some of hoenderink results. In the second class, the authors assume that they have several views of the silhouette of an object and therefore several occulding contours. The work of Giblin and Weiss [GW86] falls in that class: they consider the two-dimensional case, analog to figure 2 and model the object as the envelope of its tangents. Even though they work in this simplified setting and have only done simulations we consider they work as seminal to ours. The work of Basri and Ullman [RSR8] is more practical: they show the interest of computing the magnitude of the image currature of the sillhouette of an object. It is possible. from there using a small number of viewer-centered models of the objects to predict their new appearance from any given viewpoint. Their work is restricted in the sense that they use orthographic projection for the camera and make very special assumptions on the motion of the object between the different views. Blake and Cipolia $[B C 90]$ suppose that they obscrve the surface using a camera that they describe with a spherical model and that this camera moves continously with a known motion (the motion is computed by following markings on the surface). They parametrize the surface with respect to the are length along the extremal boundary and the time. They find the computation to be very sensitive to noise on the measurement of the motion and chose to compute differential curvature rather than "urvalure. Differential curvature is the difference between two radii of curvatures. Finally liun and Binford [LBSS] have also worked on this problem. They use two views of the object and try to describe its surface by a cylinder whose section is a conic. None of these authors solve the problen of identifying ocching contours in the image.

In light of all this. our approach has several specificities which we think are interesting:

1. we identify occluding contours from triplets of images by estimating the radius of curvature of a special planar cross-section of the object surface: the radial curve (defined in section 5.1 ).

2. on the identified occluding edges, we detect the parts which are image of points on the object with zero gaussian curvature (parabolic points).

3. we model the object as the envelope of its tangent planes and use the Gauss map (defined in section 3.2) to compute the depth. the normal and the second fundamental form of the surface along the occluding edge (except at the parabolic points where the Gauss map is degenerate). 
4. we use a general camcra model that uses perspective projection and is not restricted 10 orthographic projection.

Our paper is then organized as follows. In the first part, we present the theoretical framework of our algorithns, we briefly describe the main characteristics of the experimental setup used. In the second part, we present a method for detecting and reconstructing the extremal boundaries. The third part is devoted to the study of the computation of the first two fundamental forms of the surface in the neighbourhood of the extremal boundary. We also study a special case: the observation of parabolic points and we introduce an interesting relation between the gaussian curvature and the curvature of the image curve. In the last part, we present results on real and synthetic data and discuss their accuracy.

\section{Preliminaries}

\subsection{Camera Model}

We assume that after calibration our cameras can be accurately modeled as pinholes. The important features of sucli a sistem are the optical center and the retina plane where the image is formed. Calibration tcchinicues parameters have been extensively discussed in the literature [FTS6,Tsa86].

\subsection{Object Models}

We suppose that we are looking at a smooth olject. i.e.. whose surface is at least $C^{2}$. As discuss in the introduction, for a given position of the camera, we can draw the optical rays tangent 10 the surface of the object. These rays cut a curve on the retinal plane. the sccluding contour. and touch the object along a smooth curve on its surface, the rim.

\subsection{Experimental setup}

As shown in figure 3 our system is a stereo rig. with three cameras.

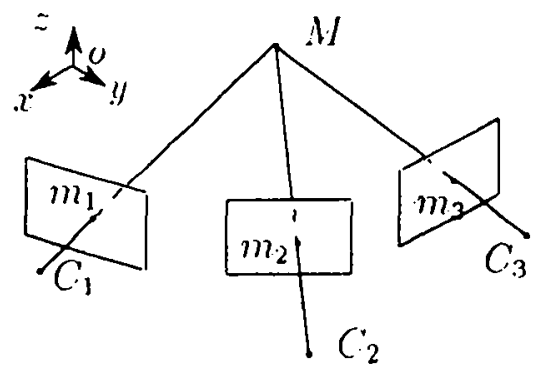

Figure 3: Trinocular stereovision system

The method that we have developed can he applied to more general systems. There are ouly two restrictions:

- We need at least threc images from a scene. The viewpoints must not be too different. With more than three images, the algorithms can be used in the same way and ricld improved results. 
- The relative positions of the different viewpoints must be known cxactly.

For the detection of edges in the images, we use a recursive implementation of the (anny filter [Ders7], followed by linking and polygonal approximation. The whole process is described in [VDF89].

\section{Computing differential properties of the object along the rim}

Like Giblin and Weiss. we consider the surface of the object as the envelope of its tangent planes but make no assumption about the camera motion or about orthographic projection on the retina plane. In fact we deal with the full perspective projection case.

\subsection{Definitions and notations:}

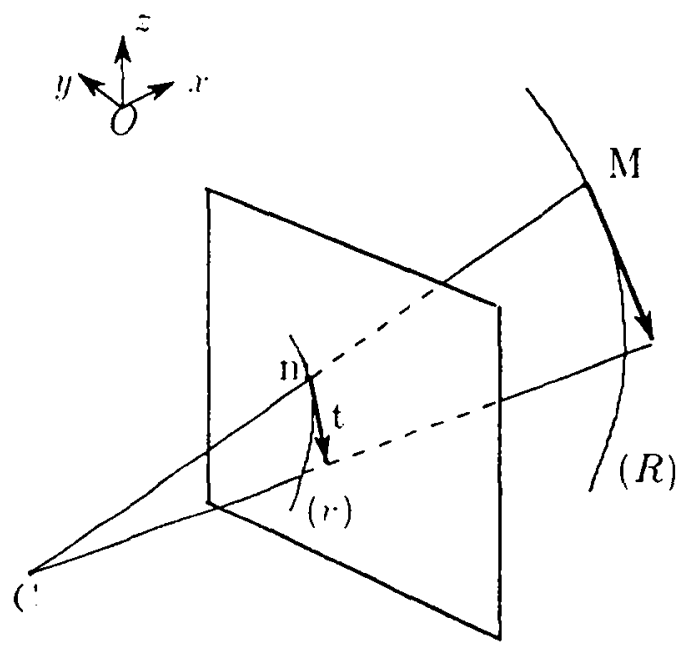

Figure l: $1 \operatorname{Rim}(R)$ and its image $(r)$

As shown in figure 4 , we consider a fixed coordinate system $(O x y z)$; the optical conter is at $C$. The camera looks at the rim $(R)$ which produces the occluding contour $(r)$. A point $m$ on $(r)$ is the image of a point $M$ on $(R)$ at which the optical ray determined by $C m$ is tangent 10 the object surface. The tangent plane to the surface at $M$ is defined by the optical ray and the tangent $t$ to the occhuling contonr at $m$. Let $n$ be the unit length normal vect or to this plane. defined by jts Euler angles $\theta$ and $\phi$ and $\nu(\theta, \delta)$ the distance from the origin to the tangent plane. The equation of this planc can be written as:

$$
n(\theta . \phi)^{T} X-\mu(\theta . \phi)=0
$$

where $X$ is the vector $(x, y, z)^{T}$ and $n=(\cos (\theta) \cos (\phi), \sin (\theta) \cos (\phi), \sin (\phi))$.

With respect to image measurements.

$$
n(\theta, \phi)=\frac{C \cdot m \wedge t}{\|C \cdot m \wedge t\|} \text { and } \mu(\theta, \phi)=n(\theta, \phi) \cdot \mathbf{O C}
$$


The observation of an occluding edge immedially yields the normal 10 the object (differemial property of order 1).

\subsection{A parametrization of the surface and the envelope theorem}

lu this paragraph. we establish that under some hypothesis, $(\theta, \phi)$ is a parametrization of the surface $(\Sigma)$ in the neighbourhood of a point $\mu$. We consider the (iauss map :

$$
N:(\Sigma)-S^{2}
$$

where $S^{2}$ is the unit sphere of $R^{3}$. To each point $p$ of $(\Sigma), N$ associates the point of $S^{2}$ whicre the normal to $(\Sigma)$ pierces the Ciauss sphere.

Theorem 1 The Gauss map is singular if and only if $h_{y}(p)=0$.

$f_{g}(p)$ is the gaussian curvature of $(\Sigma)$ at $p \in(\Sigma)$. A proof of this result can be found in [Carr6].

Now consider the mapping $(\theta, \phi)-p(\theta: \phi)$ which associates to every direction the distance from the origin to the plane tangent to the surface whose normal is in the direction $(\theta . \phi)$.

Theorem 2 For every non-paralvolic point. $(\theta, \phi)$ is " prrametrization of $(\Sigma)$ and we ran gine "parremetric equation of the surface:

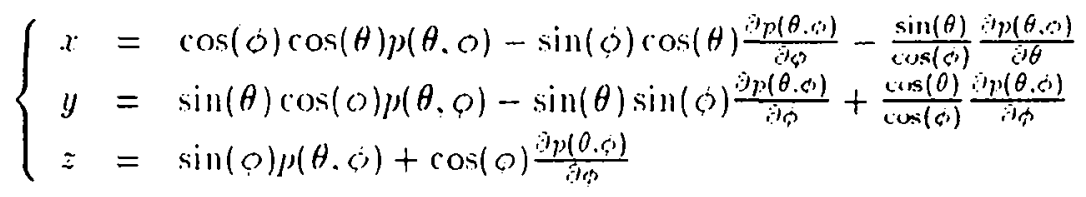

\section{Proof}

First, for every non-parabolic point, $(\theta, \phi)$ is a parametrization of the surface. We consider the following opell set of $R^{2}$ :

$$
I^{\cdot}=\{(\theta . \phi): 0<\theta<2 \pi .0<\phi<\pi\}
$$

and the map $x$

$$
\mathrm{x}: \quad \begin{aligned}
1 & \vdash R^{3} \\
(\theta \cdot \dot{\delta}) & \vdash(\cos (\theta) \cos (\phi) \cdot \sin (\theta) \cos (\phi), \sin (\phi))
\end{aligned}
$$

$\mathbf{x}(1)$ is a subset of $S^{2}$ and $\mathbf{x}$ is a parametrization of this subset. It is clearly differentiable and the jacobian matrix is

$$
\left|\begin{array}{cc}
-\sin (\theta) \cos (\phi) & -\cos (\theta) \sin (\phi) \\
\cos (\theta) \cos (\phi) & -\sin (\theta) \sin (\phi) \\
0 & \cos (\phi)
\end{array}\right|
$$

The determinant of this jacobian is null if and only if $\cos (\phi)=0$. For $S^{2}$, this occurs at the points $(0,0,1)$ and $(0,0,-1)$. Except for these points $x$, is a parametrization of $S^{2}$. The previous result on the Gauss map inplies that except for the parabolic points, $\theta, \phi$ is a parametrization of the surface. The specious point on the sphere $S^{2}$ can be eliminated by choosing an appropriate frame.

The following part of the proof uses $(\theta, \phi)$ as a paramatrization of $S^{2}$. In fact we call use any other parametrization of the sphere as for example the stereographic projection. 
We denote $\mathcal{X}(\theta, \phi)$ the parametrization of the surface (U) with the angles $(\theta, \phi)$ :

$$
\begin{aligned}
& x: \quad r \quad-(\Sigma) \\
& (\theta, \phi) \vdash \mathcal{N}(\theta, \phi)
\end{aligned}
$$

The following equation must be satisfied by the points of $(\Sigma)$ :

$$
n(\theta, \phi) \cdot X(\theta, \phi)-p(\theta, \phi)=0
$$

We derive the equation (3) with respect to $\theta$ and $\phi$.

$$
\begin{aligned}
& \frac{\partial n(\theta, \phi)^{T}}{\partial \theta} \cdot X(\theta, \phi)+n(\theta, \phi) \cdot \frac{\partial \mathcal{X}(\theta, \phi)}{\partial \theta}-\frac{\partial p(\theta, \phi)}{\partial \theta}=0 \\
& \frac{\partial n(\theta, \phi)^{T}}{\partial \phi} \cdot X(\theta, \phi)+n(\theta, \phi) \cdot \frac{\partial \mathcal{X}(\theta, \phi)}{\partial \phi}-\frac{\partial p(\theta, \phi)}{\partial \phi}=0
\end{aligned}
$$

$\frac{\partial \cdot X(\theta, \phi)}{\partial \theta}$ belongs to the tangent plane of the surface. Consequently $n \cdot \frac{\partial X(\theta, b)}{\partial \theta \theta}=0$. Also, we have $n \cdot \frac{\dot{\theta} X(\theta, \phi)}{\dot{d} \phi}=0$. The equations (4-5) turn out to be:

$$
\begin{aligned}
& \frac{\partial n(\theta, \phi)}{\partial \theta}^{T} \cdot x(\theta, \phi)-\frac{\partial p(\theta, \phi)}{\partial \theta}=0 \\
& \frac{\partial n(\theta, \phi)}{\partial \phi}^{T} \cdot x(\theta, \phi)-\frac{\partial p(\theta, \phi)}{\partial \phi}=0
\end{aligned}
$$

(1). (6) and (7) form a system of three linear equatious for the points of (I). The physical interpretation of this is that the point $M$ where the plane of equation (1) is tangent to the surface is obtained as the intersection with the planes defined by equations $(6)$ and $(i)$. We rewrite these equations in matrix form:

$$
A x=B
$$

We note that:

$$
A=\left[\begin{array}{ccc}
\cos (\theta) \cos (\phi) & \sin (\theta) \cos (\phi) & \sin (\phi) \\
-\sin (\theta) \cos (\phi) & \cos (\theta) \cos (\phi) & 0 \\
-\cos (\theta) \sin (\phi) & -\sin (\theta) \sin (\phi) & \cos (\phi)
\end{array}\right] \text { and } B=\left[\begin{array}{c}
\nu(\theta, \phi) \\
\frac{\partial p(\theta, \phi)}{\partial \theta} \\
\frac{\partial p(\theta, \phi)}{\partial \phi}
\end{array}\right]
$$

We know that the Euler angle parametrization is singular for $\cos (\phi)=0$ since all the values of $\theta$ are possible. Assuming thus $\cos (\phi) \neq 0$, we rewritc $(\$)$ ass

$$
A^{\prime} X=B^{\prime}
$$

where

$$
A^{\prime}=\left[\begin{array}{ccc}
\cos (\theta) \cos (\phi) & \sin (\theta) \cos (\phi) & \sin (\phi) \\
-\sin (\theta) & \cos (\theta) & 0 \\
-\cos (\theta) \sin (\phi) & -\sin (\theta) \sin (\phi) & \cos (\phi)
\end{array}\right] \text { and } B^{\prime}=\left[\begin{array}{c}
p(\theta, \phi) \\
\frac{1}{\cos (\phi)} \frac{\partial p\left(\theta, \phi^{\prime}\right)}{\partial \theta} \\
\frac{\theta(\theta, \phi)}{\theta^{\prime}(\phi)}
\end{array}\right]
$$

The matrix $A^{\prime}$ is orthogonal $\left(A^{\prime} A^{\prime}=I\right)$ and thus we have: 


$$
X=A^{\prime \prime} B^{\prime}
$$

This ckefincs $X$ as a function of $\theta$ and $\phi$.

\subsection{Computation of the second order differential properties of the object shape}

In this part, we show that it is possible to compute the two main curvatures of the surface for a point $M$ of the extremal boundary of the surface. $\Lambda$ remarkable point is that this compulation needs only second order derivatives of the image measurements.

\subsubsection{Some useful equations}

We have previously established that if we observe an occluding boundary and that if we suppose that $(\theta . \phi)$ is an admissible parametrization of the surface in the neighborhood of $H$. a point located on the rim, we can obtain $X=X(\theta, \phi)$.

The two fundamental quadratic forms of a surface can be derived from this expression. They require the computation of the following expressions:

- $\frac{\partial(0.0)}{\partial \theta} \cdot \frac{\partial x(0.0)}{\partial \theta}$

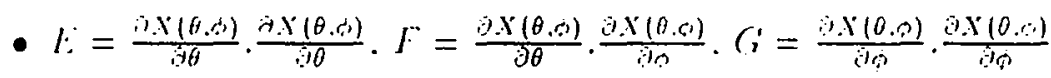

- $\|=\sqrt{1:\left(i-F^{2}\right.}$

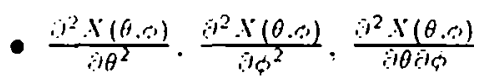

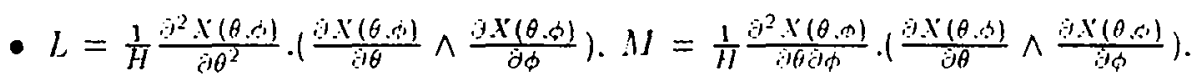

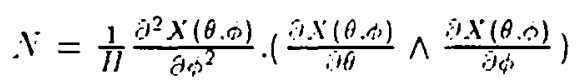

The expressions of the first two fundanental cuadratic forms are:

$$
\begin{aligned}
& \Phi_{1}\left(\lambda \frac{\partial X(\theta, \phi)}{\partial \theta}+\mu \frac{\partial X(\theta, \phi)}{\partial \phi}\right)=E \lambda^{2}+2 F \lambda \mu+C \mu^{2} \\
& \Phi_{2}\left(\lambda \frac{\partial X(\theta, \phi)}{\partial \theta}+\mu \frac{\partial X(\theta, \phi)}{\partial \phi}\right)=L \lambda^{2}+2 M \lambda \mu+N \mu^{2}
\end{aligned}
$$

The values of the two principal curvatures and principal directions are easily obtajned from these expressions by finding the eigenvalues of the endomorphism associated to $\Phi_{2}$ (detailed in B). All these derivatives can be computed by using the analytical expression that we have found in (10). We have derived them with the help of a svmbolic coniputation packinge and the equations are presented in appendix $B$. 


\subsubsection{The necessary elements}

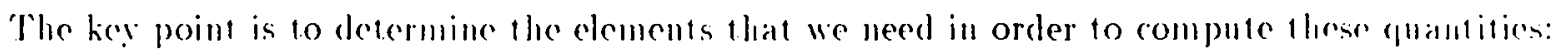

- $X=X(\theta . \phi)$ depends on $\theta . \phi, \mu(\theta . \phi)$ and the first derivatives of $\nu(\theta, \phi)$ with respere $10 \theta$ and $\phi$.

- The first derivatives of $X(\theta, \phi)$ depend on the second derivatives of $p(\theta, 0)$.

- The second dejiatives of $f(\theta, \phi)$ depend on the third derivatives of $p(\theta, \delta)$.

- Fortunately, the third derivatives disappear in the expression of $L, M$ and $N$.

After these computations we obtain that the evaluation of the first and second fundamemtal quadratic forms requires an estimation of the value of $\theta, \phi, p(\theta, \phi), \frac{\partial p\left(\theta, \phi_{\theta}\right)}{\partial \theta}, \frac{\partial p\left(\theta, \phi_{1}\right)}{\partial \phi}: \frac{\theta^{2} p(\theta, \theta)}{\partial \theta^{2}}$. $\frac{\partial^{2} p\left(\theta . \phi^{(j)}\right)}{\partial \phi^{2}}, \frac{\partial^{2} p(\theta, \phi)}{\partial \theta \dot{\partial} \phi}$. This is very interesting since these values can be estimated with sufficiently: good accuracy for the points belonging to an extremal boundary, as shown later.

Note: This property is somewhat similar to the 2-D case when we try to compute lie curvature of an arc which is defined as the envelope of its tangent lines.

\subsection{General description of the reconstruction process}

We have written the main eguations. The problem is that they involve derivatives and we wish to use them in the discrete case: we have only three images to estimate all the cuantities. The differents steps of the computation will be:

- Detect the extremal boundary and match them among the images.

- Compute $p(\theta .0)$ and its derivatives:

Mathematically. there are no difficulties; it is in practice that they arise. Indend we meanure pieces of the surface $[\theta, \phi . \mu(\theta . \phi)]^{T}$, the perlal surface $(\mathcal{F})$ from which we have to estimate first and second order derivatives which in turn vield properties of the object surface.

How is this estimation carried out? Returning to figure 4 and assuming that the curve $(r)$ in the retina plane has been identified as an occluding contonr (ways of achieving this identification are explained later in sections 4 and 5$)$. if we move point $m$ along $(r)$. the tangent plane varjes in a predictable manner and we obtain, in general. a piece of curve drawn on the pedal suface (figure j). If we move the camera a bit and assume that we know its motion. we observe anolber occluding contour and generate another piece of curve on $(\mathcal{F})$.

For a given point $M_{i}$ on the rim $(R)$ defined by the values of $\theta_{i}, \phi_{i}$ and $p\left(\theta_{i}, \phi_{i}\right)$. if we can obtain sufficiently many curves on the surface $(\mathcal{P})$ in the vicinity of $\left[\theta_{i}, c_{2}, p\left(\theta_{i}, \sigma_{i}\right)\right]^{T}$ by moving the camera with sufficient accuracy then we may hope that the first and second order derivatives of $p$ can be accurately estimated and therefore that $M_{i}$ can be reconstructed and the lifferential properties of the object surface at $M_{i}$ computed.

In the following sections, we will investigate robust ways of achieving such a goal.

Note: The Gauss-Bonnet theorem if you choose 6 functions: $E, F, C$ and $J, M, N$ lhese equations are necessary and sufficient conditions such that there is a surface ( $\Sigma$ ) having these functions as coefficients of its first and second fundamental form. They are differential cepuations and imply the first and second derivatives of $E, F, C$ and $L, M, N$. Consequently. the third order derivatives of $p$ are needed. As we do not produce any estimate of these guantities. 


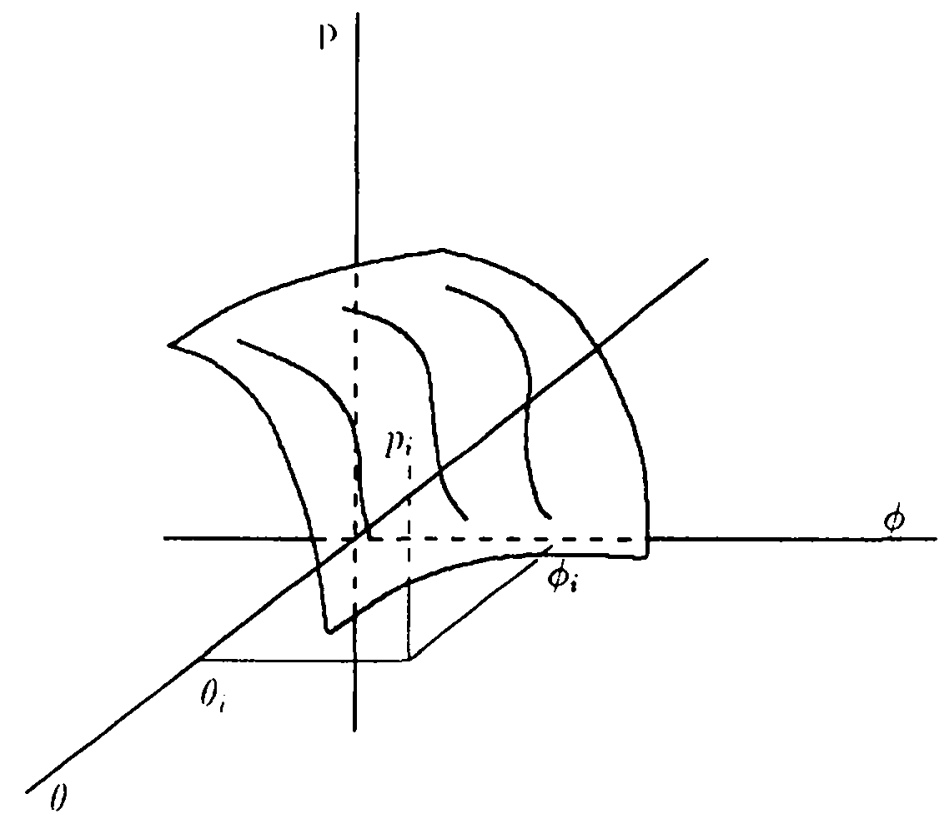

ligure 5: The pedal surface $(\mathcal{F})$

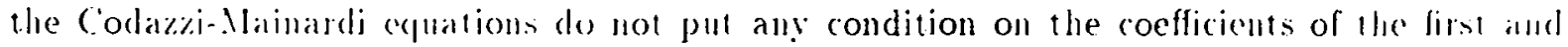
second fundamental form that we compute. In fact, they can be used as constraint equations for computing the third order derilatives of $\mu(\theta .0)$ or equivalently the second order deritatives of $X(\theta, 0)$.

\section{A first try at the detection of occluding contours: the cylin- drical case}

In the previous part. We have assumed that we can detect the extremal boundaries. In lact this is not an eas! problem. Wo show in this part that sophisticated models are needed. We first assume a simplified model in which we surface to be locally cylindrical along the rim. 'l'his investigation is interesting as it provicles us with some ideas about the mumerical stabiliv of the problem aud gain a better understanding of the difficulties. Futhermore it is used as a building block of the more general algorithm described in section 5 .

In the following sections. We present our notations. some of the techniques which can be usind to detect extremal boundaries but which suffer from some major defects and the technigue that we propose.

\subsection{Notation}

As shown in figure 6 . We consider a fixed coordinate system $(O x y z)$ with the optical conler at $(\therefore$ The camera looks at the rim $(R)$ which produces the occluding contour $(r)$. We assume that the indage curve can be reasonably approximated by using polygons. Conseguently lino occluding contour $(1)$ is a line segment $(p /)$ which is the image of a $3 D$ line $(P Q)$. For each point $m$ belonging to the occluding contour (1). the optical ray determined by ("m is tangent 10 


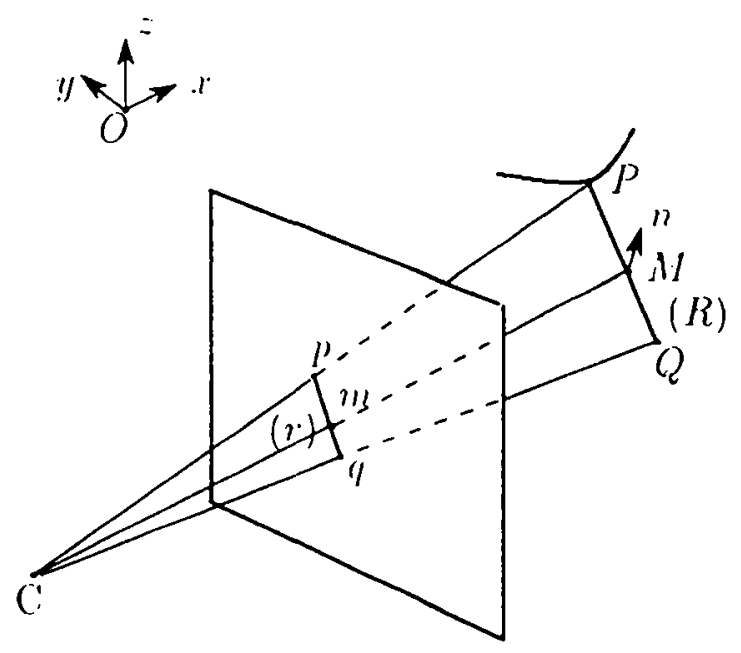

Figure (i: $A R \mathrm{Rm}(R)$ and its image $(r)$

the surface. The tangent plane to the surface at $M$ is clefined by the optical ray and the vector $m$. Let $"$ be the unit length nomal vector to this plane. and $p$ the distance from the origin to the tangent plane. The equation of this plane can be writen as:

$$
n^{\top} X-p=0
$$

where $X$ is the vector $[x, y \cdot z]^{T}$.

\subsection{Detection by the violation of the epipolar constraint}

One of the jdeas which can be used for the detection of the extremal boundary is the violation of the epipolar constraint. If you consider figure 7 . the points $m_{1}$ and $m_{2}$ are the images of two different physical points: $/ I_{1}$ and $/ Z_{2}$. As a consequence they do not respect the epipolar constraint: i.e.e $m_{2}$ does not belong to the plane $\left(c_{1} . C_{2}, m_{1}\right.$. This property secms to be an interesting characteristic and it is possible to quantify it by measuring the distance between the point $m_{2}$ and the plane $\left(C_{1} \cdot C_{2} \cdot m_{1}\right.$. Nonetheless there are wo objections to this method:

- It works in the proposed case because we suppose that we have detected the boundary of the object. Consecuently. each of the image points $m_{1}$ and $m_{2}$ is defined as the intersection of two lines. One of these lines is the extremal boundary and the other is obtained by intersection of wo surfaces: the first one is the smoolh surface corresponding to the extremal boundary and the second one is the planar surface which cuts the other surface. This is a special case. In general, we delect image-segments with poor precision at their end points. It is not safe to use the distance between these points and the epipolar plane as it depends strongly on the edge detection process.

- 'This method requires setting a threshold and as we show hater. this is quite difficult because the threshold depends on the curvature of the observed object. 


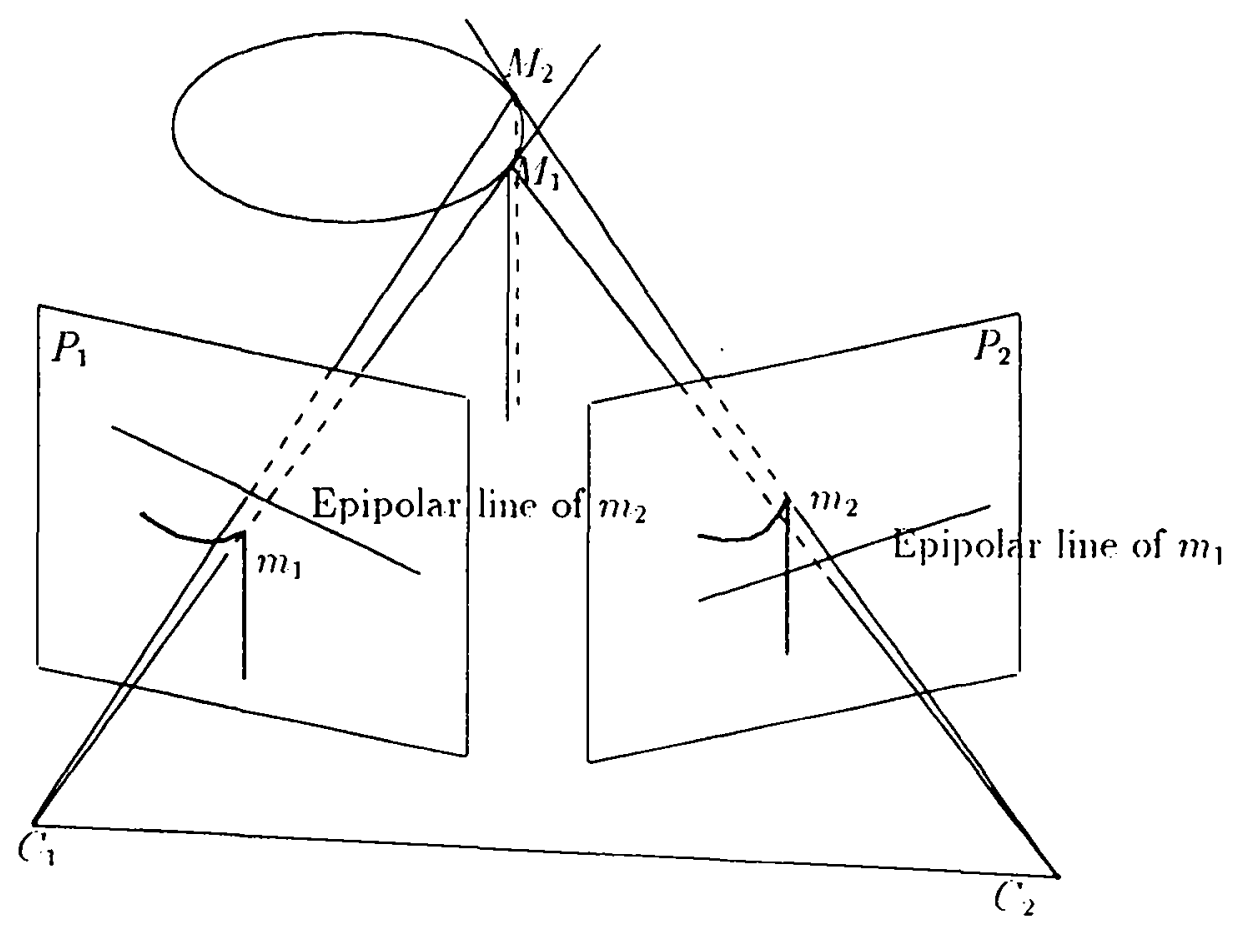

Figure 7 : Violation of the rpipolar coustraint

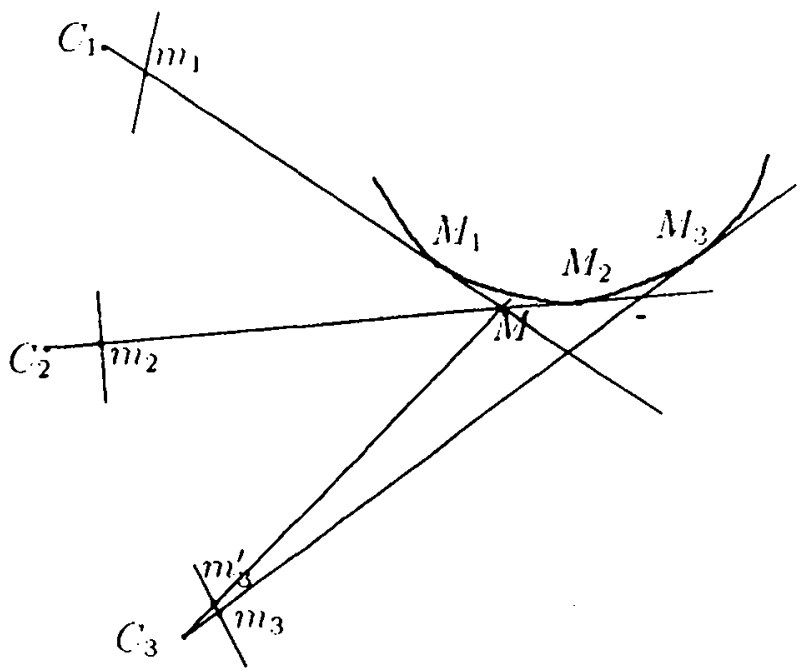

Figure 8: Detection by using motion 


\subsection{Detection by using motion}

Since oceluding edges do not correspond to phesical properties of the surfare but depend on the vicwpoint. their position on the surface changes according to the viewpoint (figure s). This means that when observing an extremal boundary with two cameras. the points observed on the surface in each camera are not the same. let us suppose that we have matched some poimts $m_{i}, i=1 \ldots 3$ between the three images. It we select two of them, $m_{1}$ and $m_{2}$. we can compule the position of the corresponding $3 \mathrm{D}$ point $M$.

We can then compute the image $m_{3}^{\prime}$ of $M$ in the third camera. It is dear that if $m_{3}$ and $m_{3}^{\prime}$ are significantly different. then the observed edge is an extremal boundary.

This approach uses directly the most characteristic property of extremal boundaries. It suffers from one problem, as in the previous method in that we have to fix a threshold to decide if the considered edge is an extremal boundary. It is difficult to cloose this threshold as the distance between $m_{3}$ and $m_{3}^{\prime}$ depends on the distance between the cameras and the object and the orientation of the extremal boundary with respect to the triangle formed by the optical centers of the three cameras and on the curvature of the surface near $1 /$.

As an example figure 9 represents the variation of the distance between $m_{3}$ and $m_{3}^{\prime}$. for an object which is rotating in front of the system of cameras. The axis of rotation is perpendicular to the plane formed by the three optical center. The object is a synthetic cylinder. Each of the three curves correspond to the reconstruction with a pair of cameras and the reprojection on the third. The horizontal axis characterizes the motion of the object. In this case. it is an angle.

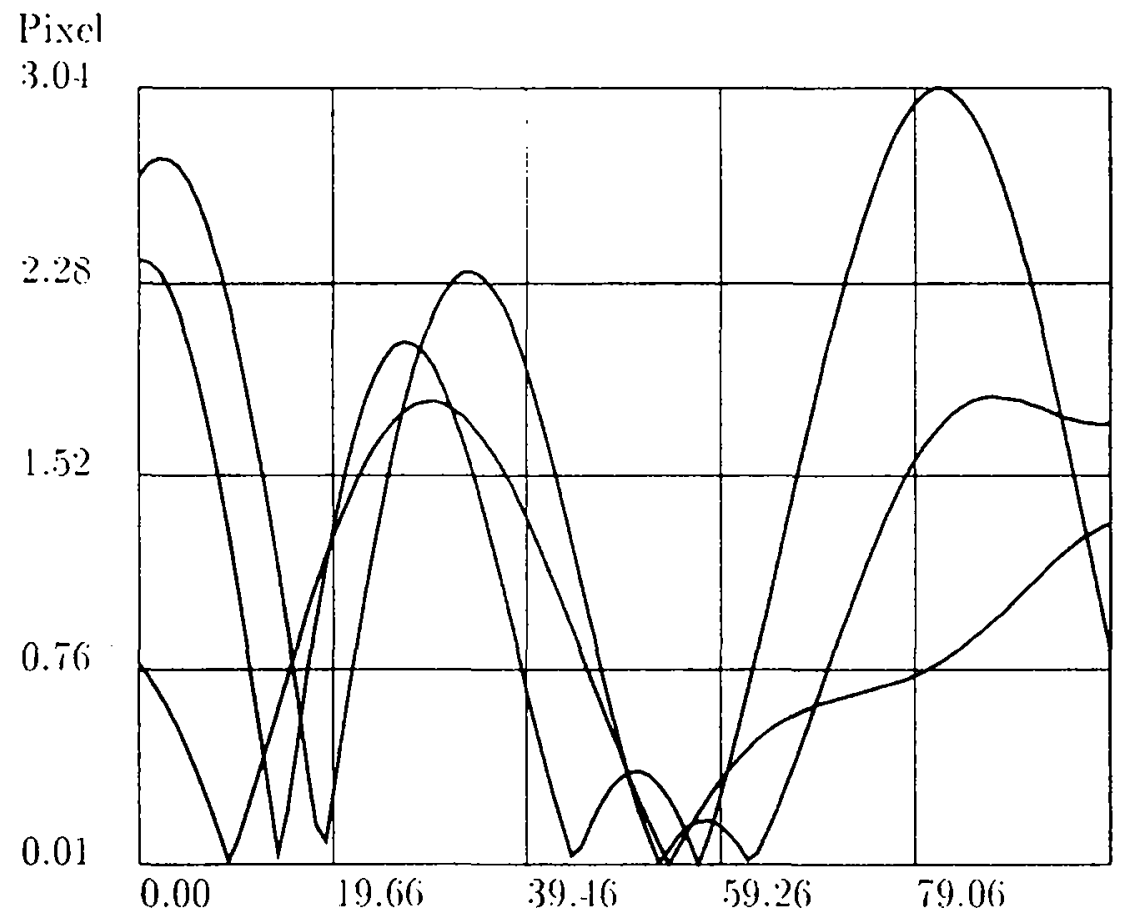

Figure 9: Rotational motion

Figure 10 represents the variation of the distance for a cylinder which has a transhational motion. The distance between the cameras and flir cylinder increases. The distance heneren 
$m i_{3}$ and $m_{3}^{\prime}$ decreases.

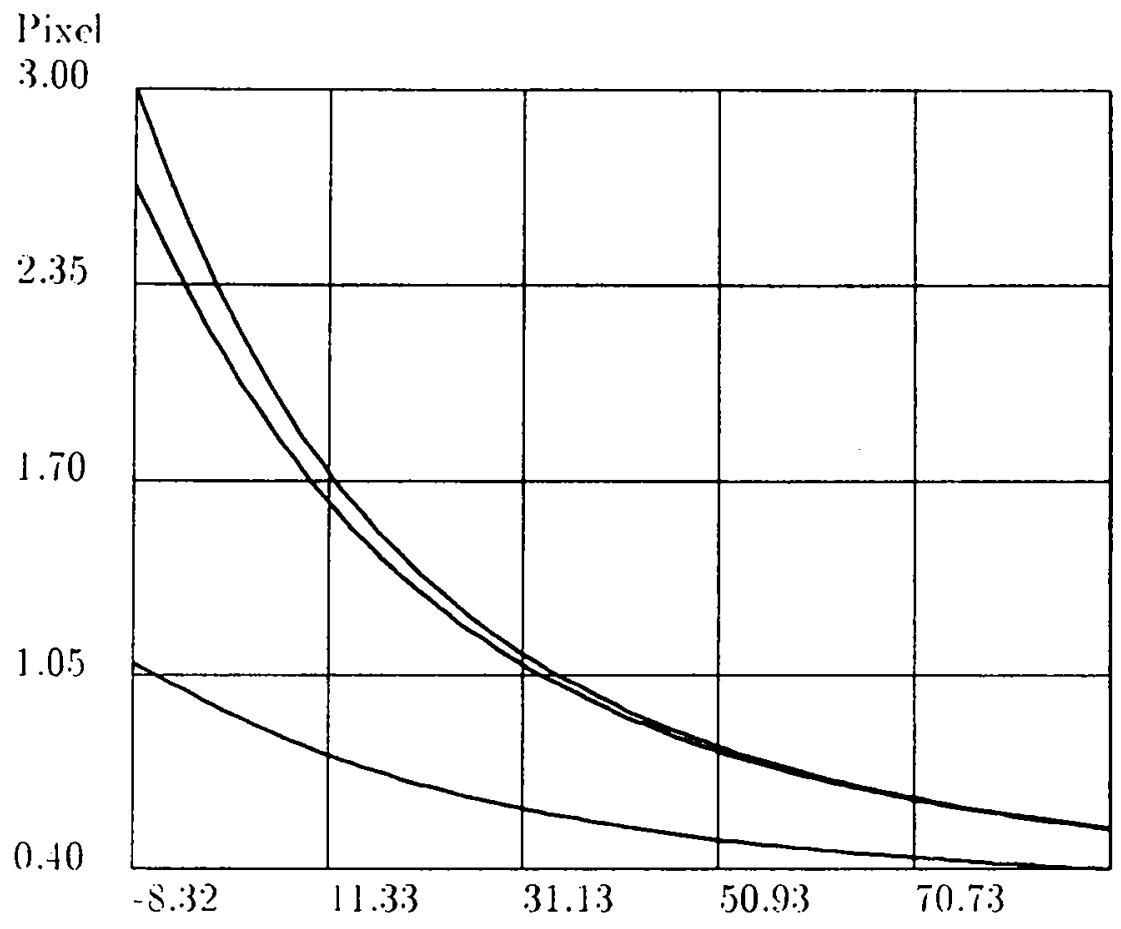

Figure 10: Translational motion

Figure 11 is the variation of the distance between $m_{3}$ and $m_{3}^{\prime}$ for a cylinder which has increasing radius. The interval of variation is $[0.001,3.0]$ pixels when the cylinder has a constant malius. In the last case. the maximum value is more than 12 pixels!

The small values are obtained when the extremal boundary is parallel to the epipolar plate. This parameter varies cnormonsly, which shows that we need a fairly sophisticated model for tracking 1 hese varations and detecting the extremal boundary.

\subsection{Detection using a cylinder model}

We suppose that we lave matched segments between different images. We want to verify if the'? belong to an extrenal boundary. One way to proceed is to assume that they do belong to one and to write the corresponding equations. We make the hypothesis that the observed surface is part of a cylinder. This provides us with a number of equations that can be used to compute the parameters of the hypothetical cylinder: its axis and its radius. Fortunately this computation can bo divided into two indepondent parts:

- the direction of the axis of the cylinder.

- the position of the axis and the radius of the cylinder.

\subsubsection{Computation of the direction of the axis of the cylinder}




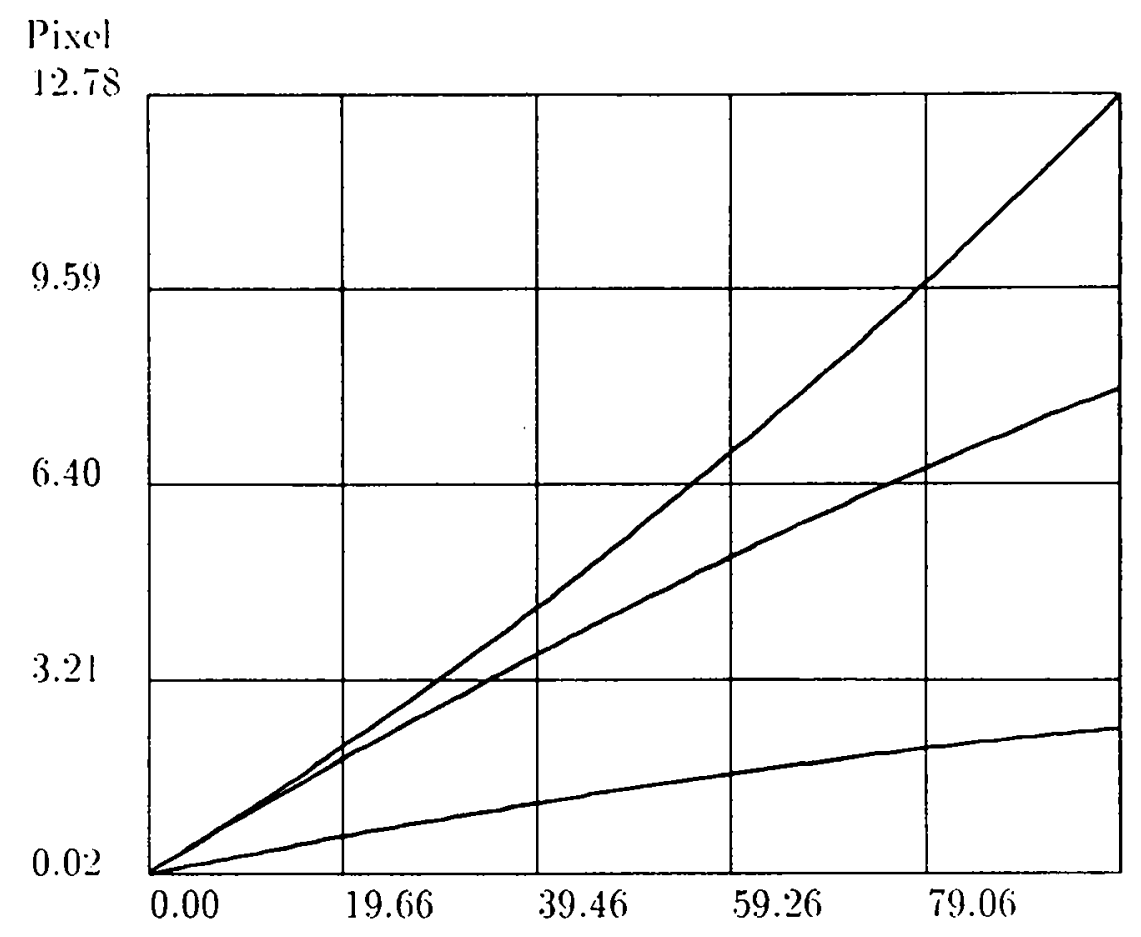

Figure 11: Increasing radius

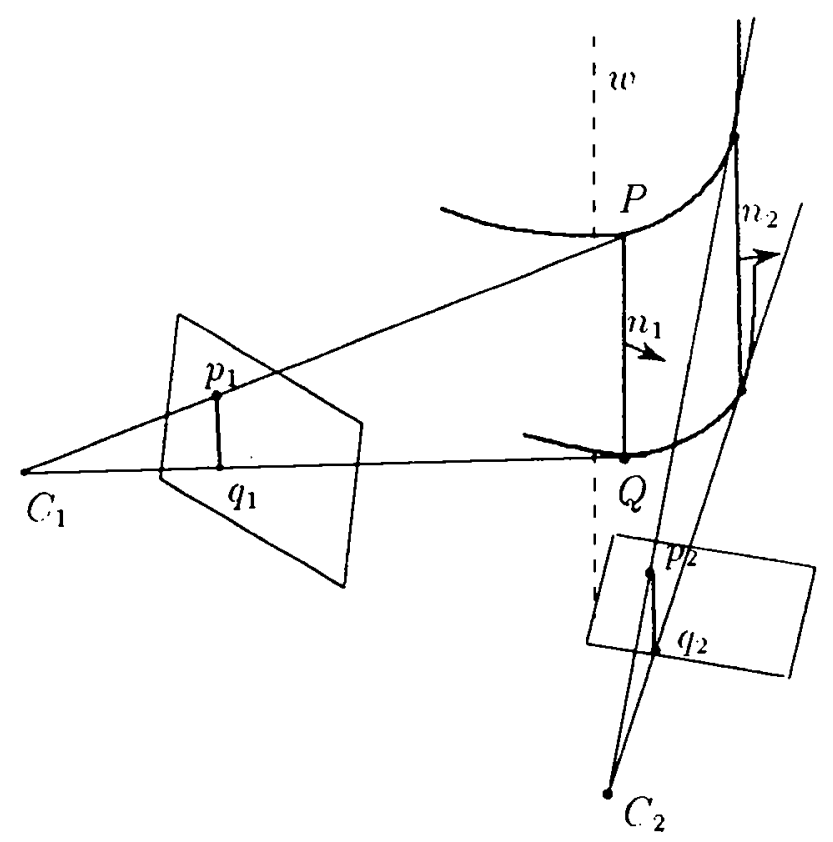

Figure 12: Computation of the axis of the cylinder 
The image of the rim of a cyliuder is a line. We can consider the optical plane corresponding to the image linc. We have defined it in (1). Let us call $w$, the direction of the axis of the cylinder (figure 12).

The line $(P Q)$ is a generatrix of the cylinder which means that it is parallel to the axis of the cylinder. This line belongs 10 the optical plane and it yields the direction $w=n_{1} \wedge n_{2}$ of the reconstructed $3 \mathrm{D}$ line.

\subsubsection{Computation of the position of the axis and the radius of the cylinder}

These computations are very simple if we perform them in the right coordinate system. $A$ good one is (Ourw). where $w$ is the direction of the axis of the cylinder, $u$ and $v$ define an arbitrary frame in the plane II which is perpendicular to $w$. The projection of the cylinder is a circle $C$. In the exact case, the line $P Q$ must be parallel to the vector $w$, the points $P$ and $Q$ must have the same orthogonal projection on the plane $\mathcal{P}$ and the projection of the optical plane must be a line. In fact unless there are only two cameras. the line $P Q$ is not exactly perpendicular to the plane $I I$ as we have computed $w$ with a minimization technique.

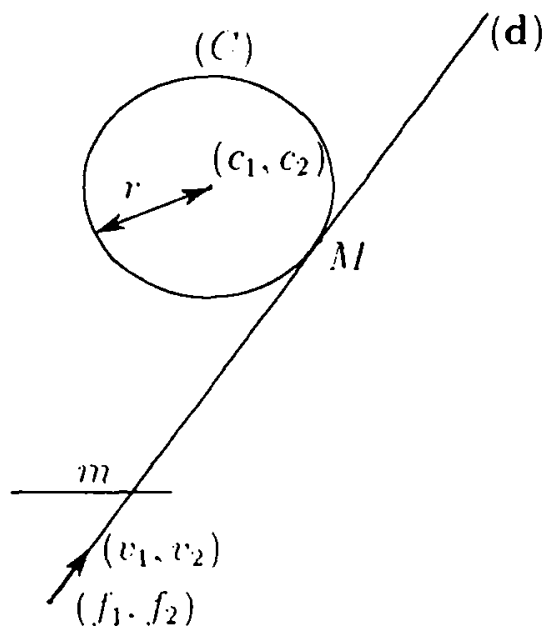

Figure 13: Computation of the center and the radius of the cylinder

Let (d) be the projection of the optical ray. The coordinates of the support vector are written $\left(l_{1}, v_{2}\right)$. Let $\left(f_{1}, f_{2}\right)$ be the coordinates of the projection of the optical center of the camera on the plane $\Pi$ and $\left(c_{1} \cdot c_{2}\right)$ the coordinates of the center of the circle $\mathcal{C}$ and $r$ its radius. The projection of the optical ray is tangent to the circle $\mathcal{C}$. This yields the following equation:

$$
d\left(\left(c_{1}, c_{2}\right),(\mathbf{d})\right)=r
$$

This equation can be rew ritten as a linear equation:

$$
f_{1} c_{2} r_{2}-f_{2} c_{1}-c_{1} c_{2}+c_{2} c_{1} r_{1}-r=0
$$

where $e$ is a variable whose value is 1 or -1 . It depends on the position of the circle with respect to the line (d). 


\subsubsection{The number of solutions}

The number of solutions depends on the number of cameras:

- For two cameras. llace is an infinity of solutions. The figure 14 is an illustration of such a situation.

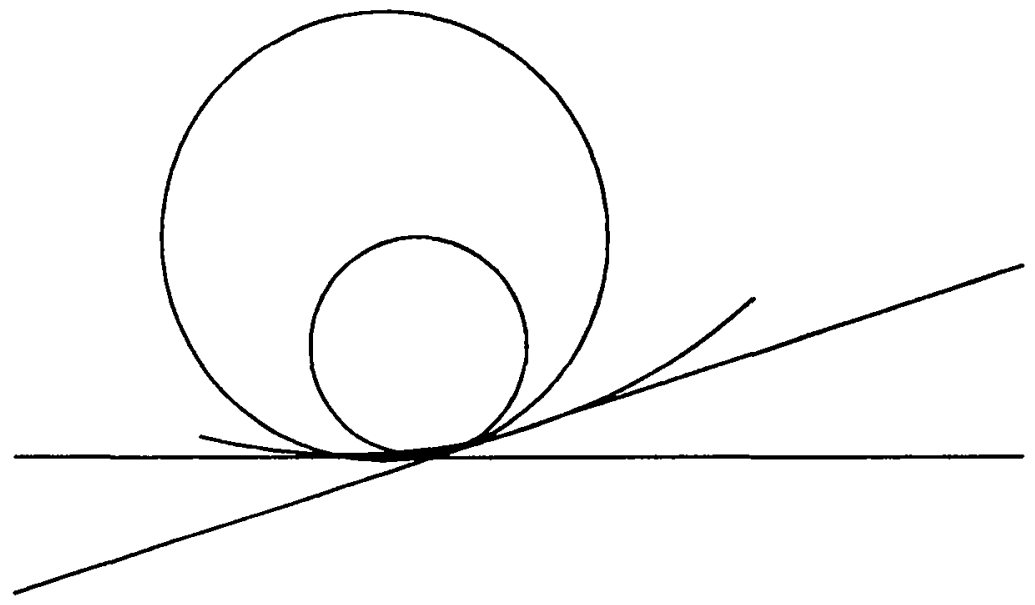

Figure 1.4: Two rameras

- For three cameras. there are exactly four solutions. They are shown in figure 15.

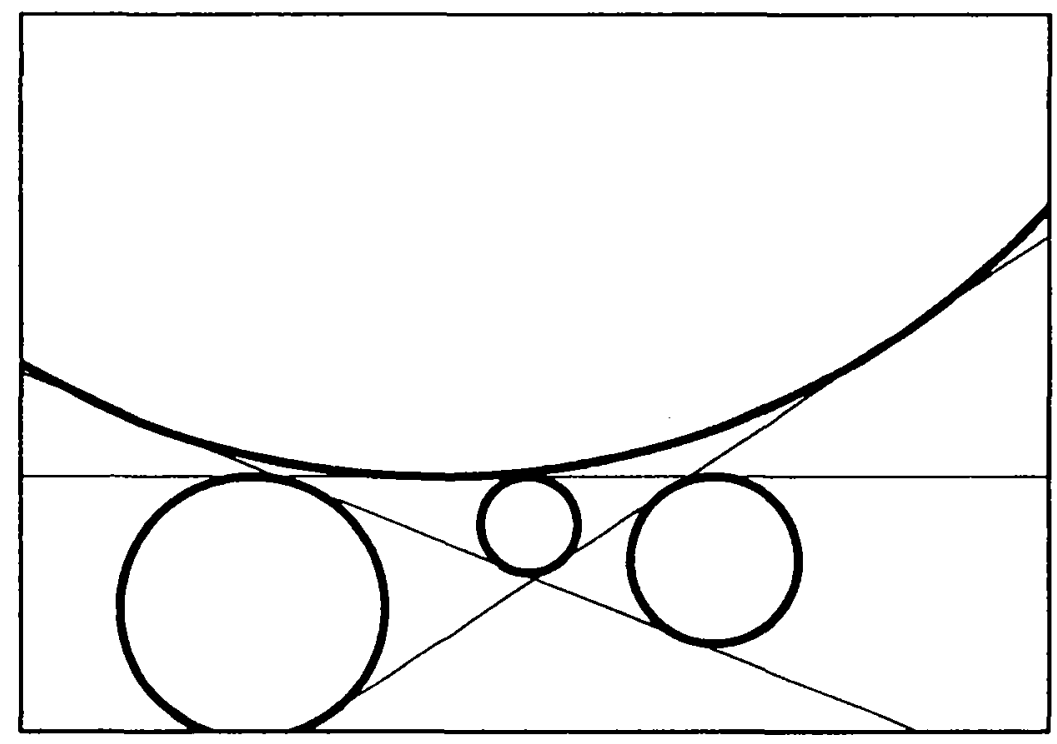

Figure 15: Three cameras. For clarity of the figure, the distances between the optical cemers have been increased 
- For more than three cameras. there are no solutions in the general case.

This result proves first that we need at least three cameras to detect an occluding edge. The method of detection by motion that we lave proposed gives the same minimal number. The first method needs only two cameras because it uses a supplementary hypothesis that we have a point to point match.

The system of equations is solved with least-squares techniques and with all the differeut sets of value possible for $\epsilon_{i}$. The correct solution can be obtained by using a physical critcrion. As we have made the assumption that the viewpoints are not very different, we can assert that the object must be on the same side of all the optical rays. This constraint produces a unirue circle.

We have proposed a set of equations which can be used to compute the parametcrs of a cylinder such that the observed line segments are the image of its rim, as seen fron each camera. We need a criterion to check whether our hypothesis is correct i.e. do we observe the rim of something which is locally cylindrical or a normal edge. We first note that the morlel we used is still correct if we suppose that the radius of the cylinder is zero. A cylinder of zero radius is physically equivalent to a normal edge. The occluding edges and the normal colges can be classified by performing a test on the value of the radius. There is still a problem: we liave to fix a threshold for taking a decision. Fortunately. we show in the next section that it is possible to attach a measure of confidence to the value of the radius.

\subsubsection{Uncertainty estimation}

We want to estimate the uncertainty on the measure of the radius of the cylinder. Wr can consider that we have constructed a function f such that

$$
r=f\left(u_{1}, n_{1}, \cdots, u_{n}, v_{n}\right)
$$

where $\left(u_{i}, c_{i}\right)$ are the coordinates of the extrmities of the image-segments. We suppose that these values are corrupted by a gaussian nojse of variance $\sigma_{u}$ and $\sigma_{v}$. In this case, we can express the uncertainty on $r$ by the formula:

$$
\sigma=\sqrt{\sum_{u_{i}} \frac{\partial f\left(u_{i} \cdot r_{1} \ldots\right)^{2}}{\partial u_{i}} \sigma_{u}^{2}+\sum_{v_{i}} \frac{\partial f\left(u_{i}, v_{i} \ldots\right)^{2}}{\partial v_{i}} \sigma_{v}^{2}}
$$

The expression of $f$ is

$$
\int=\left[\left(A^{T} A\right)^{-1} A^{T} B\right] ;
$$

where $A$ is a $n \times 3$ matrix and $B$ is a $n \times 1$ matrix. They are constructed with the linear equations presented in the previous paragraph. These matrices depend on $\left(n_{i}, v_{i}\right)$. Wilh the help of a symbolic algebraic reduction system, we call obtain the expression of the derivatives of $A$ and $B$ with respect to $n_{i}$ and $r_{i}$.

We can now create a good criterion to clieck if an edge is an occluding edge or not. A normal edge is characterized by a zero radius. The criterion is based on the probability for zero to be in the interval of confidence.

$$
r-2 \sigma<0<r
$$

In fact we evaluate the critcrion $c=(r-2 \sigma) / r$ and we decide that the obscrved edge is an occluding edge if $\mathcal{C}>s$ where $s$ is a threshold which indicates the probability of the assortion 
that we have chosen. As we suppose that he measures are corrupted be these ganssian uoise, s is a threshold based on the ganssian distribution.

The validity of llese computations can be verified in lwo ways

- The first is 10 use the law of large numbers. We define a svuthetic cylinder and compute its image using a set of calibration paraneters l hat we have obtained with real cameras. Then we use the image-segment to compute the parameters of the cylinder and the uncertainty of the estimation of $r$. In the last stcp, we repeat these computations a large number of times after adding gaussian nojse on the coordinates of the projected segment. The probability of the computed radius staying in the uncertainty interval is well described by the properties of the gaussian distribution.

Figure 16 gives the result of a such a sequence of lests. The curve is the distribution of the computed radius. The average value is the initial radius (the central dotted vertical line). Note that this curve looks like a gaussian curve. The two fine dotted vertical line are the values of $r-\sigma$ et $r+\sigma$. $\sigma$ is the computed standard deviation computed on the radius.

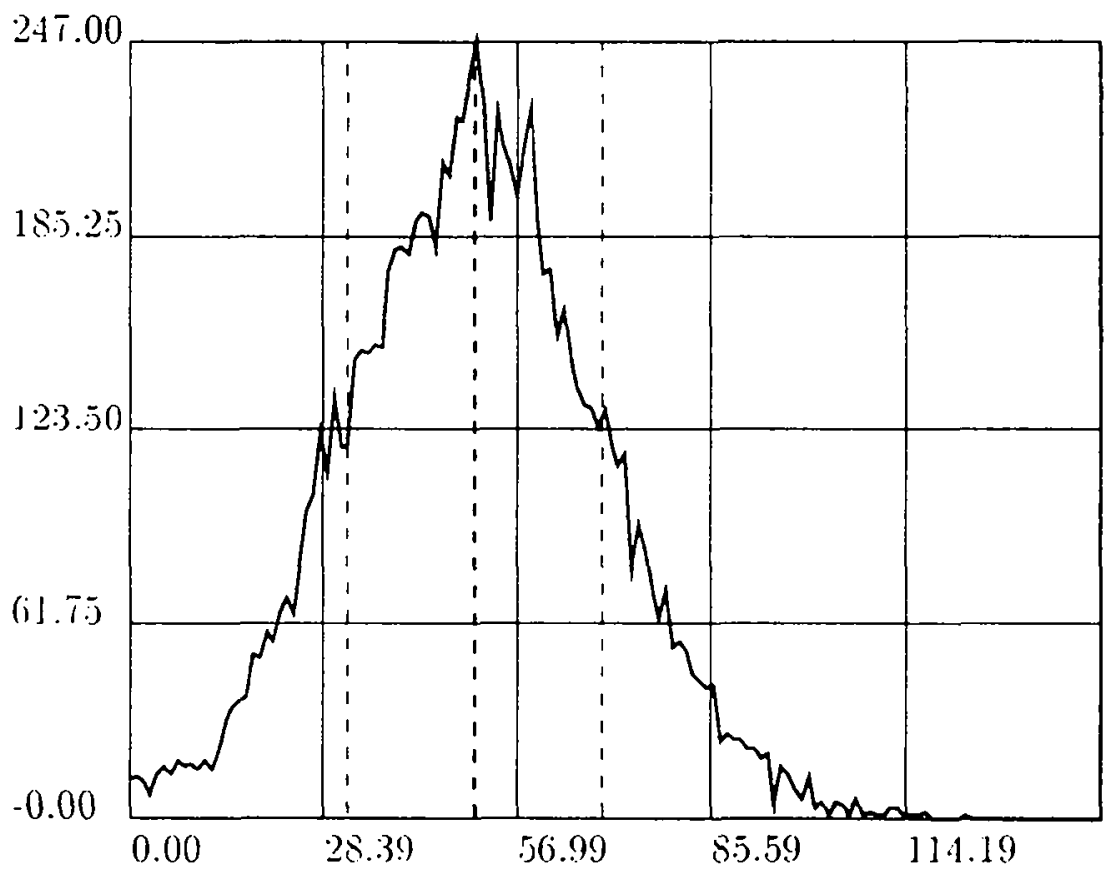

Figure 16: Statistical test on the radius

- The second is to study the variations of the uncertainty with respect to the positions of the cylinder. We can then compare the variations of the criterion with the variations of distance between the junge-segment of the cylinder and the segment which is the image of a normal edge which has the same spatial position that the occluding edge of the cylinder. We perform this test on the same symthetic data as in the study on the wariation of the distance (paragraph 4.3). The results are presented in figure 17 . 


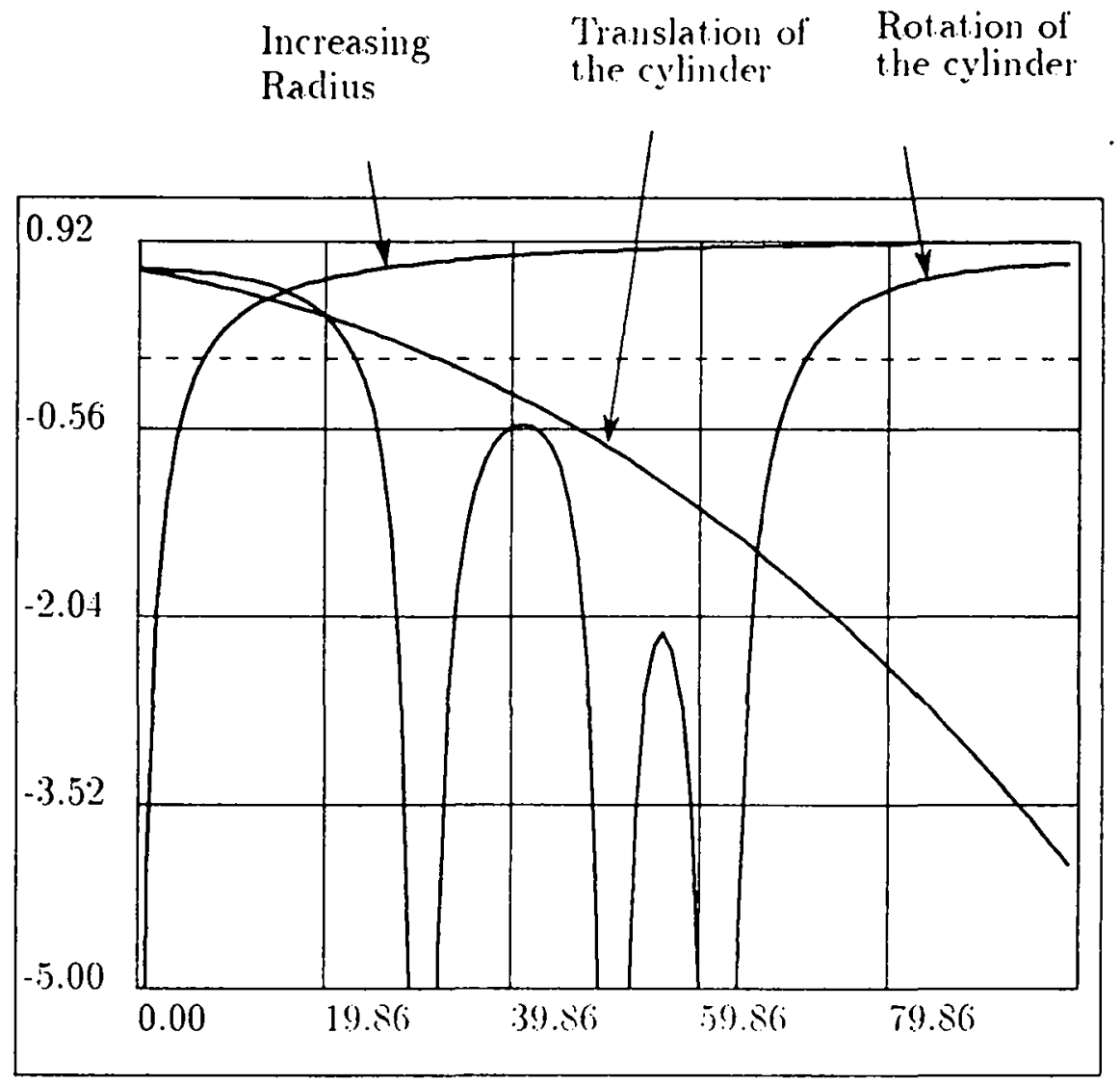

Figure 17: Variation of $\mathcal{C}$ with respect to several parameters. As the three curves do not have the same kind of abscissa, they cannot be compared. 


\begin{tabular}{|c|c|c|c|}
\hline Real Radius & Estimated Rarlius & $\sigma$ & $\mathcal{C}$ \\
\hline 0 & 1.9 & 24.5 & $<0$ \\
\hline 50 & 59.2 & 23.2 & 0.21 \\
\hline 100 & 98.3 & 22.1 & 0.55 \\
\hline 130 & 114.5 & 21.3 & 0.62 \\
\hline 160 & 17.5 .2 & 20.0 & 0.77 \\
\hline
\end{tabular}

Table J: Five șinthetic ç̣linders

- In the case of rotation, there are positions where $\mathcal{C}$ is very small. In these cases. the extremal boundary is parallel to one of the epipolar planes and it is almost inpossible to classify the contour. In the method of the paragraph 4.3 , we have exactly the same kind of problems at these points.

- In the case of translation. the criterion clecreases and it becomes impossible to detect the cylinder.

- When the radius increases. the criterion increases also.

These three factors. orientation. distance and radius are obviously linked 10 each other: for example the significative value is the distance divided by the radius.

\subsection{Results of the implemented system}

We have tested the algorithm on symthetic and roal data.

\subsubsection{Synthetic data}

The objectives of the test on syuthetic lata are

- testing the software.

- the rerification of the noise model that we have used.

The principle of these tests is to take a description of a system of real camcras and to simulate the obseriation of a cylinder. In fact. we only compute the image-segment of the extremal boundary of the segment. We add some noise to the extremities of the endpoints of this image-segment. We use a gallssian noise with a variance of one pixel.

Table 1 shows the values of the following parameters for a set of five cylinders: the radius of the cylinder. the estimated radius. the value of the uncertainty $\sigma$. the criterion $C$.

The observation is that the estimated radius always stays in the interval $[r-2 \sigma, r+2 \sigma]$. The value of $\sigma$ decreases when the adius increases. This is in accordance with our intuition: when the cylinder has a large radius the part which is observed is more important and the cylinder can be reconstructed more accurately.

The baseline is approximatively $250 \mathrm{~mm}$ and the distance from the optical center of the camcras to the objects is about 800 millimeters.

These experiments shows that our ideas are correct and that we can obtain realistic estimations of the uncertainty. Nonetheless, we have to keep in mind that we have not modeled the uncertainty on the calibration of the cameras. 


\subsubsection{Real data}

The figures the examples are showed by presenting the three original images and the reconstructed edgers. We use two technigues for showing the reconstructed edges.

- In the first way, we undesline the segments which have not been recognized as cxiremal boundaries. There are four kinds of lines:

- Dotted fine line: these segments hare not been considesed because they are too small or they have been matched in only two cameras.

- Dotted thick line: these segments have becn detected as extremal boundaries.

- Continuous fine line: these segments have been classified as normal edges with a large confidence $(C<0$ and $\sigma$ small $)$.

- Continuous thick line: these segments have becu classified as with the lower confidence that the previous $(C<0$ and $\sigma$ hig $)$.

- In the second way, we underline the segments which have been classified as extremal boundaries. There are four kind of lines:

- Dotted fine line: these segments have not been considered.

- Dothod thict line: hliese segments have heen classified as nomal edges.

- Contimous thick line: these segments have been classified as cxtremal boundaries.

- Continuous fune line: these segments ane the computed axis of the cylinders. In order to establish the connection between the axis and the extremal boundary there is a segment between these iwn.

The results We present two triplet of imagcs:

1. The first one is a scene with two cylinders and some polyhedric objects (figure 18 and figure 19). The results of the algorithm are precise for the segments which have an orientation sufficiently different from the horizontal. (The segment on the left side of the big cylinder is an crroneous matclu. This is why it is reconstructed as a cylinder with a false axis.)

2. The sccond scene includes two cylinders and a bos wilh a lot of markings (figure 20 and figure 21 ).

\subsection{Conclusion}

The main result of this section is that it is possible to detect the extremal boundaries in the case of locally cylindrical objects. We have to use a model of mocertainty 10 come up with a quantitative way of setting the decision threshold. It is clear that this process can provide false results if there are erroneous matches or if the observed occluding contonr does not correspond to a cylinder but to a more general surface. In he next section. we deal with the general casc. 


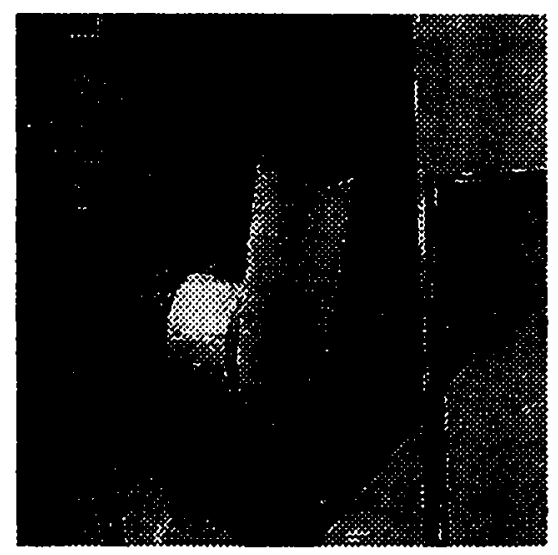

Camera 1

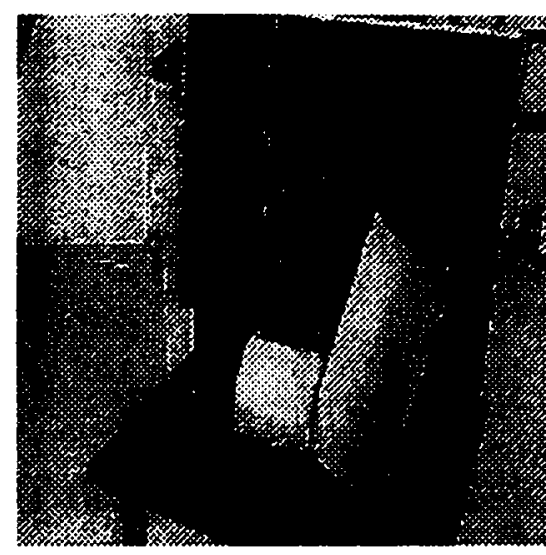

Camera 2

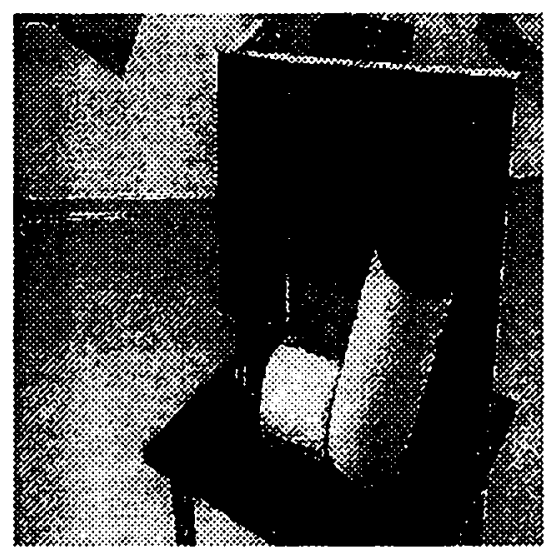

('amera:

Figure 1s: "lriplen "I" and the polygonal approximations

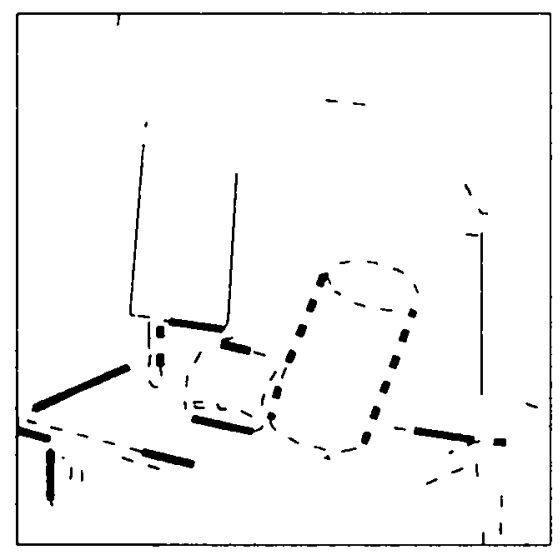

Pirst Wa!

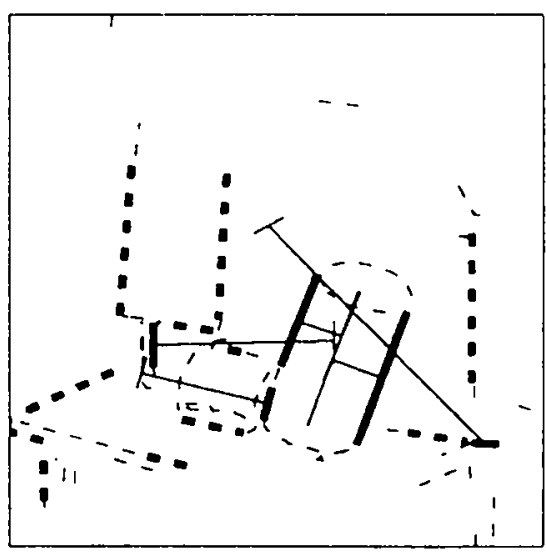

Second way:

Figure 19: The reconstructed segunents and the extremal boundaries: tripiet "I" 


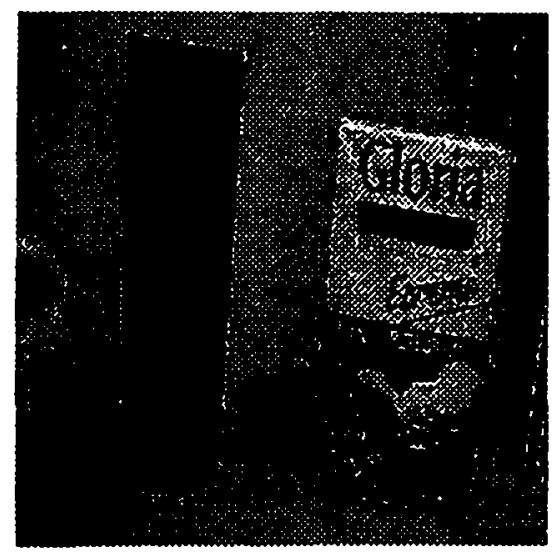

Camera 1

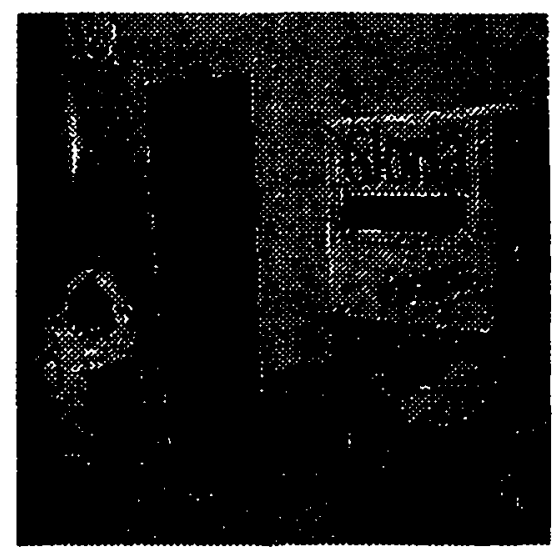

Camera 2

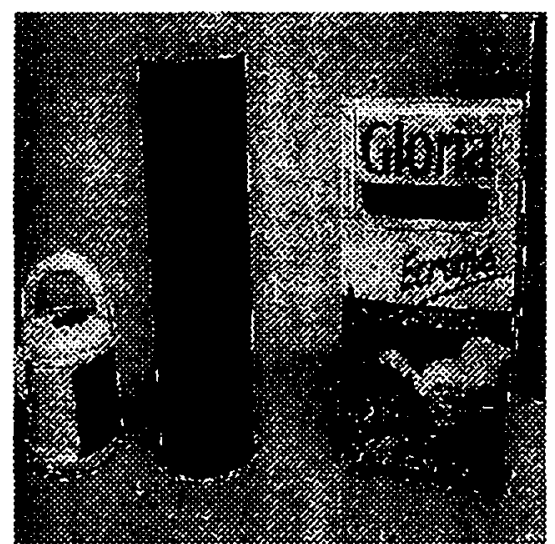

Camera :3

Figure 20: "lriplen "..2" and the polygonal approximations

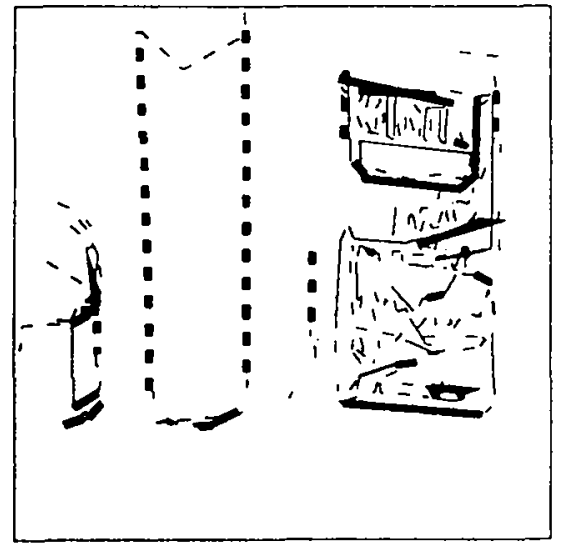

Pirst way

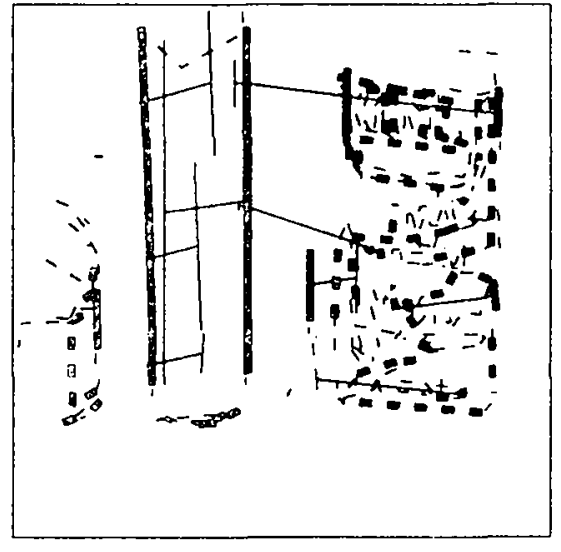

Second way.

Figure 21: The reconstancted segments and lle extremai boundaries: triplen $\cdots 2$ " 


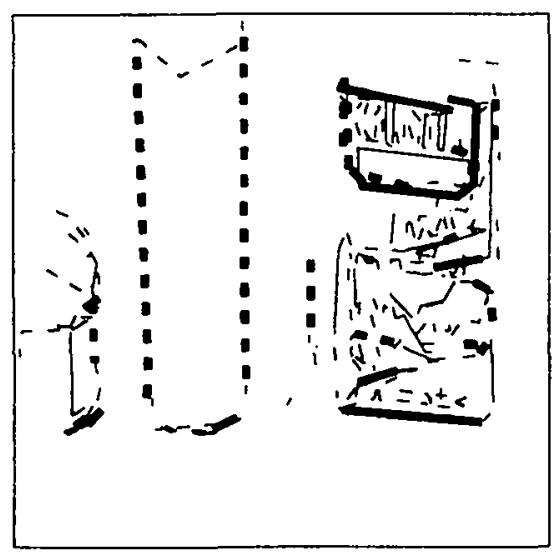

First way

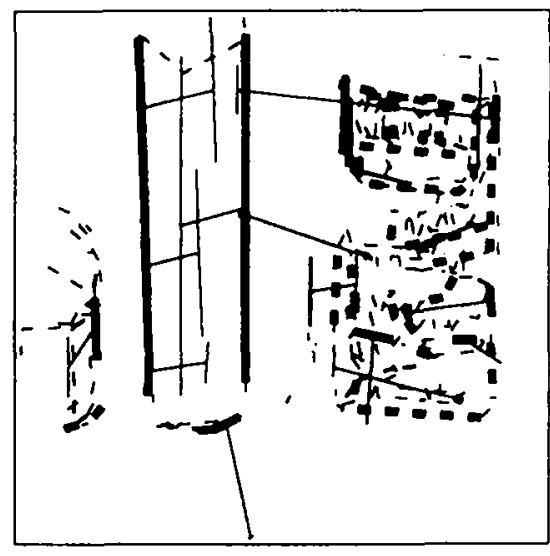

Second way

Figure 22: The reconstructed segments and the extremal boundaries: triplet "2" (with several observations)

\section{Detection of occluding contours: the general case}

In this part, we suppose that we have observed an edge from several viewpoint and we want 10 decide if it is an extrental boundary and if so to compute some properties of the surface in the neighbourthood of the rim. We are interested by the differential properties up to the second order of the surface. liundamental theorems of differential geometry [Cari6] assert that these properties are sufficiont to characterize the surface up to a rigid displacement.

- The zero order differential property is the simple estimation of the position of the point. It means that we have to compute the exact position of the contact point between the surface and the optical ray for each of the cameras.

- The first order differential property is the estimation of the tangent plane to the surface. It is the casjest to obtain as we are observing an extremal boundary. In this case the langent plane is the optical plane.

- The second order properties are the most difficult to obtain as they require the evaluation of second order derivatives. Such computation call be sensitive to noise.

There are two rechuical points which will not be discussed in detail:

1. The matching of the image curves corresponding to the extremal boundaries. We solved this problem by using a stereorision algorithm which has been described in [VF89].

2. The problem of representing the image curves. We use a spline approximation in order to have a more precise represcntation of the edges and to be able to compute the tangent. vector and the curvaluse along the points of the edges [Vaigo].

\subsection{The depth of the rim: the radial curve method}

We remember that the rim $(R)$ of a surface is a curve, and thus the image $(r)$ of the rim must. he a curve. It is always true in a gencric position. So, we can suppose that we have detected a 
curve $\left(r_{i}\right)$ in each image. For cach of these curves. it is possible to compute the tangent rectors at each of their points.

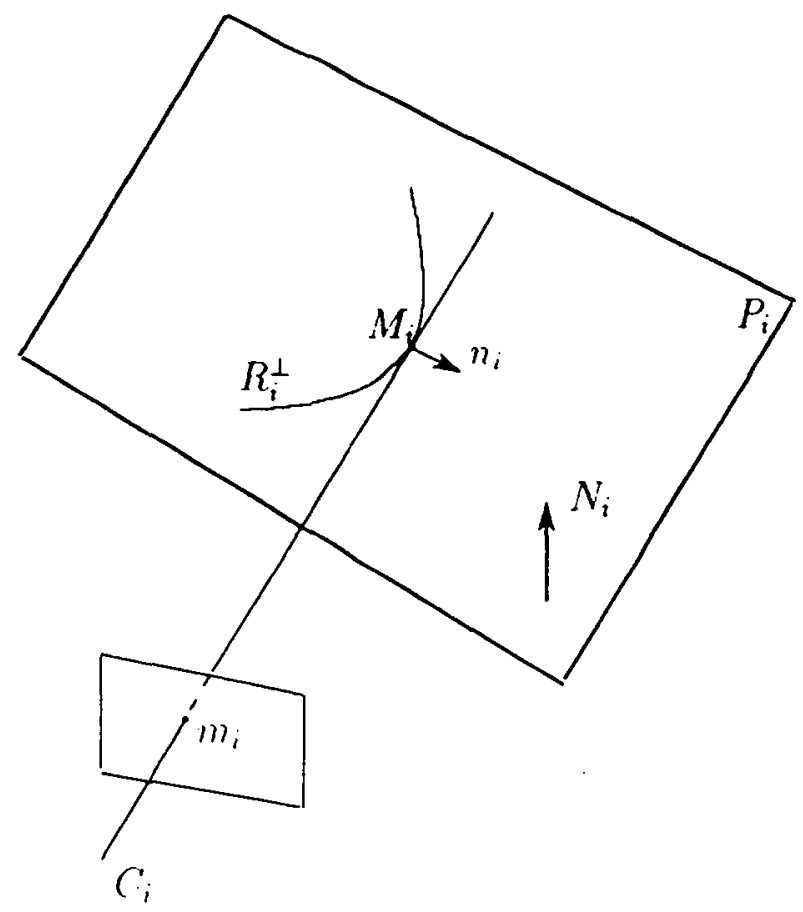

Figure 23: Tlie radial curve $R_{i}^{\perp}$

Let us choose one camera $i$ and $m_{i}$ one point on llee curve $r_{i}$. We call consider the curve $R_{i}^{\perp}$ which is the intersection of the surface of the object with the plane $P_{i}=\left(C_{i}^{\prime} N_{i}\right)$. where $C_{i}^{\prime}$ is the optical center of the camera $i$ and $\lambda_{i}=\left(C_{i} m_{i}\right) \wedge n_{i}$. $n_{i}$ is the nomal to the optical plane tangent to the surface at $M$, (figure 23 ). This plane ean be easily constructed with the langent $t_{i}$ to the occluding contour $r_{i}$. The planar curve $R_{i}^{\perp}$ is called in the literature the radial curve and its curvature is called the radial curvature $n_{i}$. The curvatule of the curve $r$ is called the apparent curvature or transterse curvature $i_{t}$.

The key idea is to neglect the apparent curvature and to use only the radial curvature. The objectives are to be able to decide if an edge is an extremal boundary or not and to compule the coordinates of the point $H_{i}$ which belongs to the surface of the object and projects 10 point $m_{i}$.

We can draw another planar curve on the surface of the object which has interesting properties. We consider the plane $E_{i, j}=\left(C_{i}^{\prime}, m_{i}, C_{j}^{\prime}\right)$ (figure 24$)$. This plane is called lice epipolas plane of $m_{i}$ with respect to camera $j^{\prime}$. Suppose that this plane intersects the jmage $r_{j}$ of the rim seen from camera $j$ at a point $m_{j}$. The jutersection of the plane $E_{i, j}$ with the surface of the object is a curve $E_{i . j}^{C}$. $M_{j}$ belongs to this curve. The epjpolar plane $E_{i, j}$ contains the optical ray $\left(C_{i}^{\prime}, m_{i}\right)$ and this implies that the point $M_{i}$ belongs also to the curve $E_{i, j}^{C}$. Horeored we can prove that $R_{i}^{\perp}$ and $E_{t, j}^{C}$ have the same tangent at the poim $M_{1}$. The tangent at $M, 10$ R $R_{i}^{\perp}$ is the optical ray by construction. The tangen at $H_{i}$ 10 $E_{, j}^{C}$ is in the plane $E_{i, j}$ by construction.

\footnotetext{
${ }^{1}$ This plane is formol by the three points m, C', and $C$,
} 


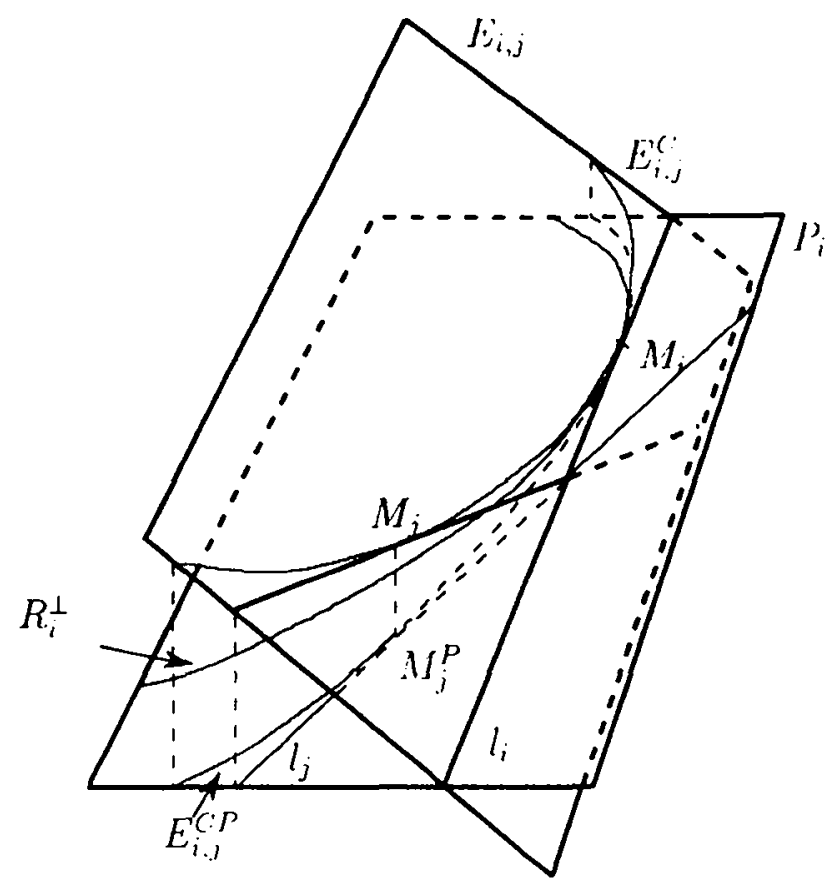

Fignre 2-: The adial curve $R_{i}^{+}$and the epipolar curve $l_{i, j}$

and in the plane tangent to the surface at $M_{i}$. as this curve is drawn on the surface. As a consequence, the tangent at $V_{i}$ to $E_{i, j}^{C}$ belongs to the following two planes: the plane langent 10 the surface at $M_{i}$ and the plane $E_{i, j}$ : The intersection of these two planes is the optical ray because the optical ray belongs to both plancs. Consequently the two curves $R_{i}^{+}$and $l_{i, j}$ have the same tangent at the point $M_{i}$. They have also the same curvature.

Now we consider the curve $E_{i, j}^{C P}$ obtained by projecting the curve $E_{i, j}^{C}$ orthogonally in the plane $P_{i}$. The tangent at $M_{i}$ to $V_{i . j}^{C} P$ is the optical ray. The tangent at the projection of $I_{j}$ 1. $E_{i, j}^{C P}$ is the projection $l_{j}$ of the optical ray $\left(, m_{, j}\right.$ on the plane $P_{i}$. If there is no apparent currature the two curves $R_{i}^{+}$and $E_{i, j}^{C P}$ will be the same. The distance between these fwo curves depends on the magnitude of the apparent curvature and the angle between the two plances. If the viewpoints are not too different. they will be very close. The idea of our method is 10 neglect the apparent curvature and to use these different projections to computate the radial rusature at. $M_{i}$.

From the coordinates of the points $m_{i}$ and $m_{j}$ and their tangemt vectors $t_{i}$ and $l_{1}$, and the calibration parameters, we can perform the following computations:

- Computation of the optical ray $\left(' ; m_{i}\right.$.

- Computation of the radial plane $P_{i}=\left(C_{i}^{\prime}, N_{i}\right)$ with $N_{i}=\left(C_{i}^{\prime} m_{i}\right) \wedge n_{i}$

- Computation of the optical ray $C_{j}^{\prime} m_{3}$ and its orthogonal projection $l_{j}$ in the plane $f_{1}$.

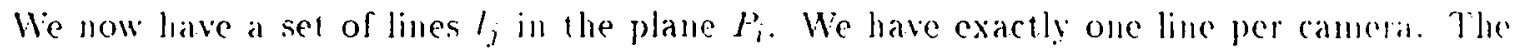
following yuestion is how to find an approximation of the radial curve and especially of the point $M_{i}$. The curve $R_{i}^{+}$is approximately tangent 10 all the lines $l_{j}$. As we have supposed that our 
viewpoints are not 100 different. the different contact proints must be neighbours and we can use these points for estimating the osculating circle to the radial curve at $M_{i}$. This is the same problem an the problem of linding the center and the radius of the cylinder in the previous part and we apply: the same techniques to solve it.

At the end. we have an estimation of the osculating circle of the ladial curve and an idea of the ralidity of this approximation. Let $r$ be its radius and $\sigma$ the vatiance. Several cases are possible:

- $0>r-2 \sigma$. It means that this part of the contour is not an occluding edge.

- $r$ and $\sigma$ are small. It means that the edge does not correspond to an extremal boundary.

- $\sigma$ is not small. It means that the geometric configuration does not pormit a good estimation of $r$ and the system of linear equations used for its computation is degenerate. For three camcras, this is the case when one of the tangent vectors $t_{i}$ is close to the epipolar line. In that case. we only have two cameras which are really usable for detecting the extremal boundary. The only condition for two image points to be the image of a unique spatial point is that they respect the epipolar constrainl. This is the case by construction of the point s $m_{i}$ so we cannot conclude anything in this situation. The only solution is to take another image by moving the objects of the system of camems.

Wir thus have a method for deciding whether or not an edge is oceluding or not. This method is basid on the computation of the radial curvature and its uncertainty.

The position of the point $M_{i}$ can then be casily deduced from the line $l$, and the paraneters of the rircte.

\subsection{The estimation of the first and second derivatives order of $p(t)$.$) .$}

The formulas that we have discussed in section 3.3 and which are given in appendix $B$ for computing the differential properties of the surfince reguire the cratuation of the first and second derivalives of $\mu(\theta . \phi)$.

\subsubsection{Estimation of the first order derivatives of $\mu(\theta, 0)$}

By applying the method described in the previous section. we can compute the coordinates of $M_{i}$. the observed point on the surface. Since we have detected that the observed edge is an extremal houndary, we can apply the equations 6 and $i$ :

$$
\begin{aligned}
& \frac{\partial p(\theta \cdot \zeta)}{\partial \theta}=\frac{\partial n(\theta \cdot \varphi)^{\gamma}}{\partial \theta} \cdot x \\
& \frac{\partial p(\theta . \phi)}{\partial \phi}=\frac{\partial n(\theta \cdot \phi)^{\gamma}}{\partial \phi} \cdot x
\end{aligned}
$$

Ho have a simple expression of the first derivatives of $\mu(\theta . o)$. 


\subsubsection{The estimation of the second derivatives of $\mu(\theta . \phi)$}

If we consicler the pedal surface $(P)$ (figure 5), we measure onc curve for cach camera. These curves are drawn on $(\mathcal{P})$. $(\mathcal{P})$ is simple since we have assumed liat locally $(\theta$. $\phi)$ is an admissible paramet rization of the object surface. The best way to estimale the second derivatives is to lit locally a surface on the measured data in the space $(\theta, \varphi, p)$. The problem is to find a good basis of functions for performing the fit. In fact, since the function $\nu(\theta, \phi)$ is periodic, it is natural to consider its fourier expansion:

$$
p(\theta, \phi)=\sum_{n, n=-\infty}^{\infty} a_{n, m} f^{i(n \theta+m(,)}
$$

where the coefficients $a_{n, m}$ satisfies $a_{n, n}=\overline{a_{-n,-m}}$.

We have limited ourselves more or less arbitrarily to those values of $n$ and $m$ satisfying $|n|+|m| \leq 1^{2}$

There are thus 13 unknown coefficients:

$$
\begin{aligned}
p(\theta . \phi)= & a_{0}+\left(a_{1} \cos (\phi)+b_{1} \sin (\phi)\right)+\left(a_{2} \cos (\theta)+b_{2} \sin (\theta)\right)+ \\
& \left(a_{3} \cos (\theta+\phi)+b_{3} \sin (\theta+\phi)\right)+\left(a_{4} \cos (\theta-\phi)+b_{4} \sin (\theta-\phi)\right)+ \\
& \left(a_{5} \cos (2 \theta)+b_{5} \sin (2 \theta)\right)+\left(a_{6} \cos (2 \phi)+b_{6} \sin (2 \phi)\right)
\end{aligned}
$$

For the minimization, we used a simple quadratic criterion and the values of $a_{i}, i=0 \ldots \ldots 6$ and $b_{i, i}=1 \ldots .6$ are obtained by inverting a $13 \times 13$ matrix. Since we have already estimated the first derivatives of $p(\theta . \phi)$. hicy an also be used in the criterion without changing the complexity.

As discussed in the introduction and in section 3.2. our method breaks down when the points on the rim are parabolic. It is therefore important to be able lo detect such points reliably. Ile discuss this issue and related ones in the next two sections.

\section{The problem of parabolic points}

\subsection{Detecting parabolic points: a qualitative approach}

The idea is to use the properties of the gauss map in order to compute the sign of the gaussian curvature of a point belonging to an cxtremal boundary.

\subsubsection{Properties of the Gauss Map}

IIe make first the following remarks:

- For every point $p$ of a surface ( $(\Sigma)$ such that $p$ is a nont-parabolic point. the gauss map is a diffeomorphism between a neighbourhood of $p$ on the surface $(\Sigma)$ and $S^{2}$, the unit splere of $R^{3}$ (paragraph 3.2 ).

- Let $\Sigma$ and $\bar{\Sigma}$ be two surfaces and $\phi:(\Sigma) \vdash(\bar{\Sigma})$ be a diffeomorphism; assume that for some $p \in(\Sigma)$, the differential of $\phi$ at $p d \phi$, is non singular. $\phi$ is oricntation prescrving at

\footnotetext{
${ }^{2}$ In the case of the occluding boundary of a splete the exact equation of $p(\theta, \phi)$ can be derived: $p(\theta, \phi)=-\cos (\phi) \cos (\theta) c_{x}-\cos (\phi) \sin (\theta) c_{y}-\sin (\phi) c_{z}-r$

where $\left(c_{1}, c_{y}, c_{z}\right)$ are the coordinates of the center and, the radius.
} 


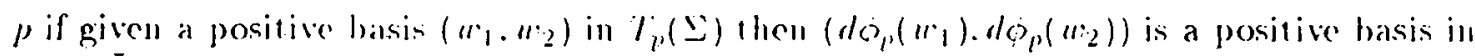
$T_{\phi(p)}(\bar{\Sigma})$.

- $(\Sigma)$ and $S^{2}$ are two surfaces of $R^{3}$. Thus, an orientation on ( $(\Sigma)$ induces an orjentation on $S^{2}$. Let $p \in(\Sigma)$ be such that $d N_{p}$ is uon-singular. Since for a basis $\left(w_{3}, u_{2}\right)$ in $T_{p}(\Sigma)$

$$
d N_{p}\left(u_{1}\right) \wedge d N_{p}\left(w_{2}\right)=d c t\left(d N_{p}\right) w_{1} \wedge w_{2}=r_{3}(p) u_{1} \wedge w_{2}
$$

the Gauss map will be orientation-preserving at $p \in(\Sigma)$ if $\kappa_{y}(p)$, the gaussian curvature is positive. If $h_{g}(p)<0$. then it is orientation-reversing.

Intuitively this means the following: the orientation of $T_{p}(\Sigma)$ induces an orientation of small closed curves in ( $(\Sigma)$ going around $p$; the image by the Gauss map of these curves will have the same as or the opposite orientation of the initial one. depending on whether $p$ is an elliptic or hyperbolic point, respectively.

More details about these propertics of the Gauss map can be found in [Carifi].

\subsubsection{Application to the case of the extremal boundaries}

If we consider a point $m_{p}$ in one of the cameras. For this point, we can compute:

- the point $M_{p}$ whose image is $m_{p}$ (section 5.1).

- The oriented normal on the surface (section 5.2.1) at $M_{p .}$. This is the image of $M_{1}$, ly the Cianss map).

We can also measure hese quantities for other points $m_{q}$. Among them we sclect those points $m_{q}$ image of $V_{q}$ such that $U_{q}$ is in the neighbourhood of $M_{p}$. These can be points on the same rim as $M_{p}$ or points which have matched with the epipolar constrajnt. The whole set of these points is used in order to build a small closed curve around $M_{p}$ on $(\Sigma)$. Ising the normals. we get the image of this curve hy the Cianss maj. It is a curve. There are three cascs:

- It has the same orientation as the original curve $=\Rightarrow r_{g}>0$.

- It has the opposite orientation of the original curre $\Rightarrow r_{y,}<0$.

- It is 110 a simple curve $\Rightarrow r_{g}=0$ (1his a consequence of the two first cases).

Remark Koenderink [lioes.t] shows that it is possible to idetemine the sign of the gatussian curvature by using only one image. IIC uses lypotheses which are different from ours: lic supposes that he knows that the image curve $(r)$ is an extremal boundary and an orientation of $(r)$, i.e. the position of the matter with respect to $(r)$. He need at least thee cameras in orile to establish these points.

\subsection{Detecting parabolic points: a quantitative approach}

We will now show in this section that if $H_{i}$ is a parabolic point then the currature of $(r)$ at $m$, is zero. 


\subsubsection{A relation between several curvatures}

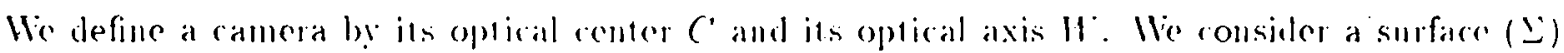
and a point $P$ on this surficr which belongs to the apparent contour of (2) We denote the transversal curvature by li, lloc curvature of $(r)$ at $p$ (image of $P$ ). We denote the radial curvature by in the curvature at $l$ ' of the curve which is the intersection of the surface and the radial plane. $f_{g}\left(f^{\prime}\right)$ is the gaussian curvature of the surface $(\Sigma)$ at $P$. We now prove the following theorem:

Theorem 3 If $r_{r} \neq 0$ \|lir"

$$
r_{i g}=n \frac{r_{t} r_{i}}{\lambda}
$$

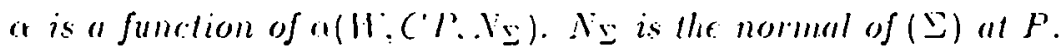

$\lambda$ is the distance between $(?$ and $P$.

If $\mathrm{ri}=0$ and $\mathrm{h}_{\mathrm{g}} \neq 0$ tho" (r) has a singular point.

The proof of this result and several rematis can be found in annex $A$.

From lheorem 3 we deduce immediately that if the point on the surface is parabolic. the'll either $n=0$, or $r_{r}=0$ or $r_{l}=0$. From equation (35), we deduce that $a$ is not zero unless $\theta=0$ and $\delta=0$. This is an impossible case as it means that $X=l: X$ is an optical ray and $l$ is a vertor of the image plane. 'Therefore:

$$
r_{! l}=0 \Rightarrow r_{i}=0 \text { or } r_{i}=0
$$

If $r_{i}=0$. the point of $(r)$ is not singular. so $r_{r} \neq 0$ and $r_{q}=0$.

$$
i_{i}=0 \Rightarrow r_{i j}=0
$$

The case $r_{y}=0$ and $r_{r}=0$ is not generic: the set of parabolic points of a generic surface is formed of curres. The set of proints betonging to $(P)$ is also formed from curves. The intersertion of these two sets is formed of points. hir $=0$ means that the optical ray has the direction of the principal direction with zero curvature (for a parabolic point the only direction with a curvature is one of the lwo principal directions). This is impossible under the general viewing assumplion.

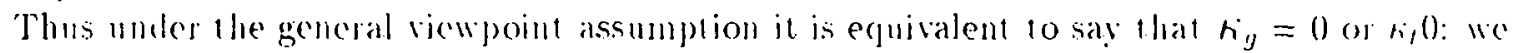
have a way to detect the parabolic points on the object from just image measurements.

Remark If we do not suppose that we are in a generic situation. strange situations call occur. Intagine that you observe a cone such that its center is equal to the optical center. The image is an ellipse $(a, \neq 0)$ eren if all the points are parabolic. Bul of course this collipse disappears if we move only slightly.

\section{Experimental Results}

We have tested those ideas on symthetic and real data and we present results with these lwo binds of data. 


\subsection{Synthetic data}

We have done tests on symlietic lata berause computing the curvalures requires calculating second order deriatives and the process of differentiation is well hnown to be noise sensitive. The of her advantage of symbetic data is that it is possible to compare the computed value to the real value.

In fact we should say "almost" symhetic since, even though we have been using syullictic models, their rims have becu projected on real 512 by 512 images and quantization noise is therefore present in the datia.

We have conducted experiments on synthetic images corresponding to a sphere, a cylinder. a one-sheet hyperboloid and a torus. We will present results on a torus as there are several interesting cases.

Figure 27 shows the surface of the torus (only the left part is visible from the cameras). 'The fine contimous lines represent the cameras.

We work in local frame $[C, u, u, w]$ such that $C$ is the optical center of the camera, " is the optical ray: $r$ is the vector normal to the tangent plane, $w=u \wedge v$, $w$ is the vector normal to the radial plane. In this local frame $\theta=\pi / 2$ and $\phi=0$. We use it because all the computations are simpler and we obtain better results. especially in the process of fitting a surface for computing the second derivatives. From cquation ( $\tilde{\tau})$, we can deduce that $\frac{\partial p(\theta, \zeta)}{\partial \phi}=0$. Figure 25 shows the values that we have computed for the first and second derivatives of $p(\theta, \phi)$. The continuous line comesponds to the romputed values. The dotted lines are the theoretical values. The aloscissa is equialent to an indice along the reconstructed chains of points. In the case of the second derivatives pant of curves are equal to zero: for these points the criterion $c$. indicates that it is impossible 10 produce a measurement as there is a problem of orientation of the epipolar plane will respect 10 the tangent of the curve.

The principal curatures are represented in figure 26.

The reconstructed edges arc drawn in figure 28 . There is one rim $(R)$ for each camera. The fine lines describe the cameras. Figure 29 represents the results that we have obtained with the qualitative approach. The continnous thick lines are the elliptic points. The dot ted thick lines are the luperbolic points. The fine continuous lines are the points which do not belong to the apparent contonr. 'They are reconstructed with a classical algorithm.

\subsection{Real data}

We present wo scenes: the first one contains a ball and the second one a bottle.

- Figures 30 and 34 are the images with the edges.

- Figures 31 and 35 represent the reconstructed chains.

- Figures 32 and 3 (j represent he principal clicetions. In the case of the ballon the principal directions vary considerably between two neighbouring points: this is because all the points are umbilical and every direction of the langent plane is a principal direclion.

- Figures 33 and 37 represent the computed radii of curvature. 'The added numbers correspond to the part of the object which is referenced respectively at the figures 32 and 36 . In figure 333. the dotted thich lines are the real walues of the radius of the ball.

- Finure 38 represents the sign of the gaussian curvalure computed with the qualitative approach in the case of the bottle. We use the sane description as for figure 29. Wie do not present these results for the ball an all the points have been found to be elliptic. 


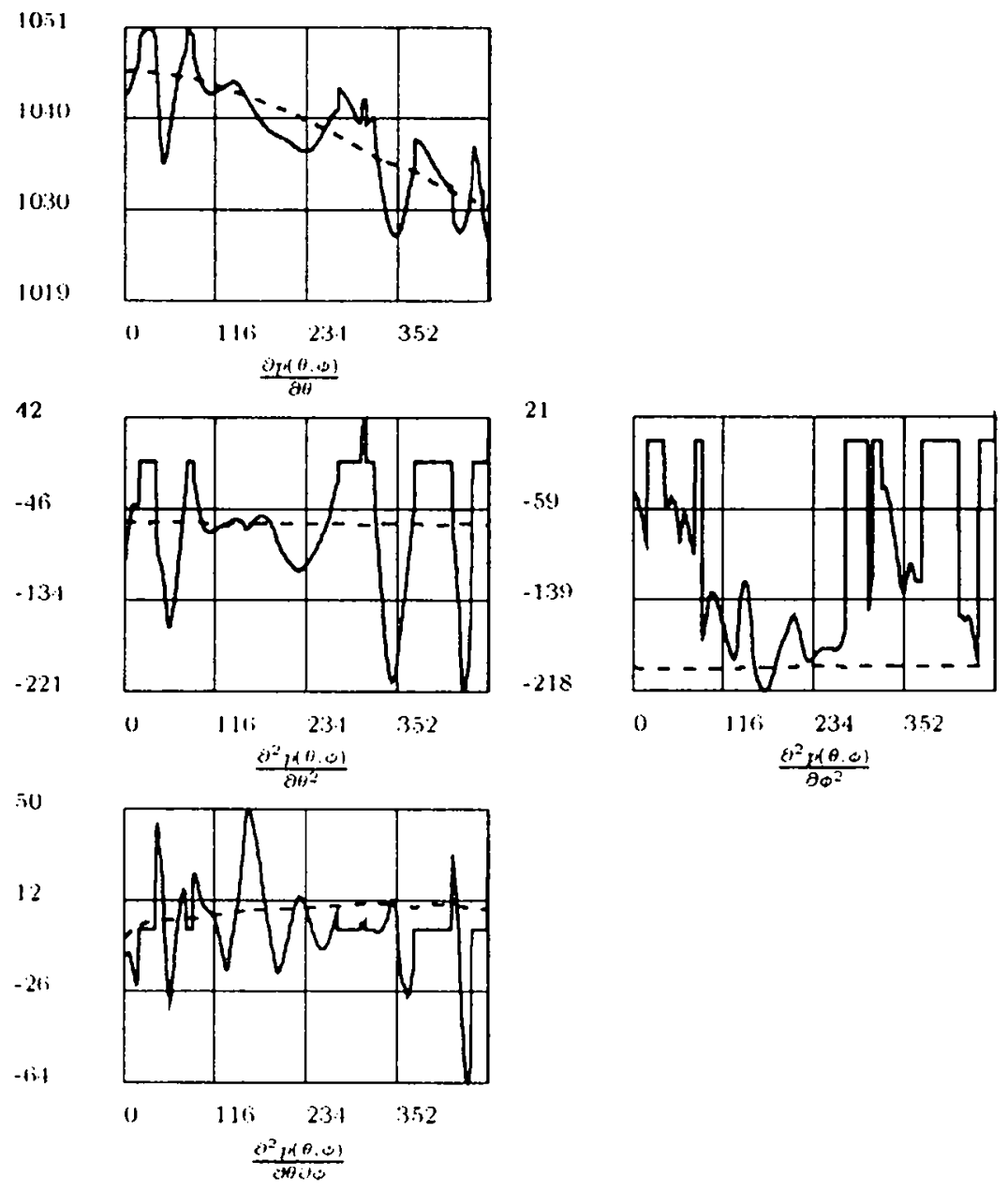

Figure 25: The deriative curves for the torus

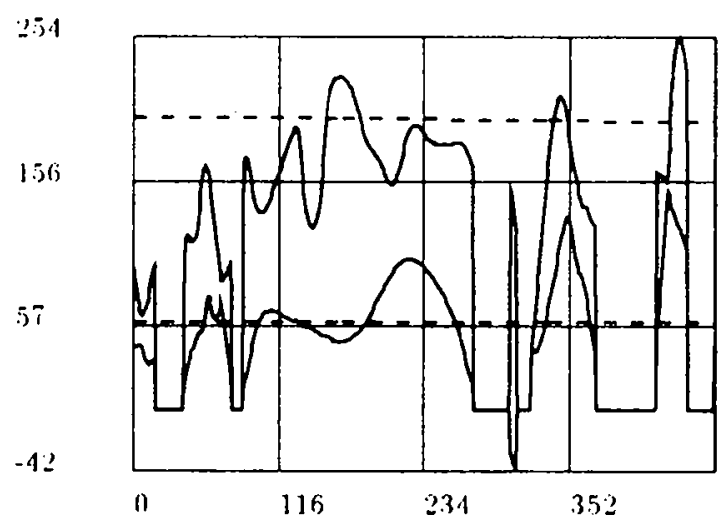

Figuse 26: The radii of curvature for the torus 


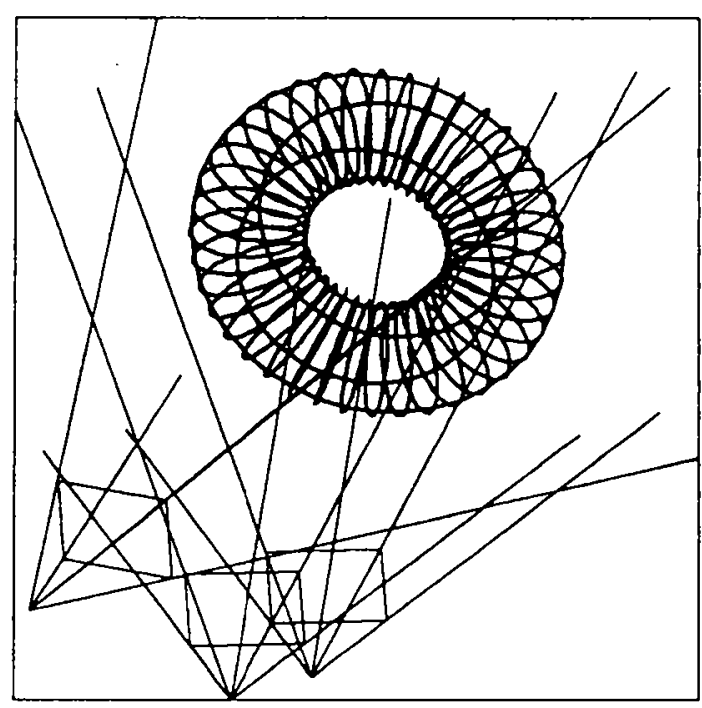

Fignere 22: The syuthetic torus

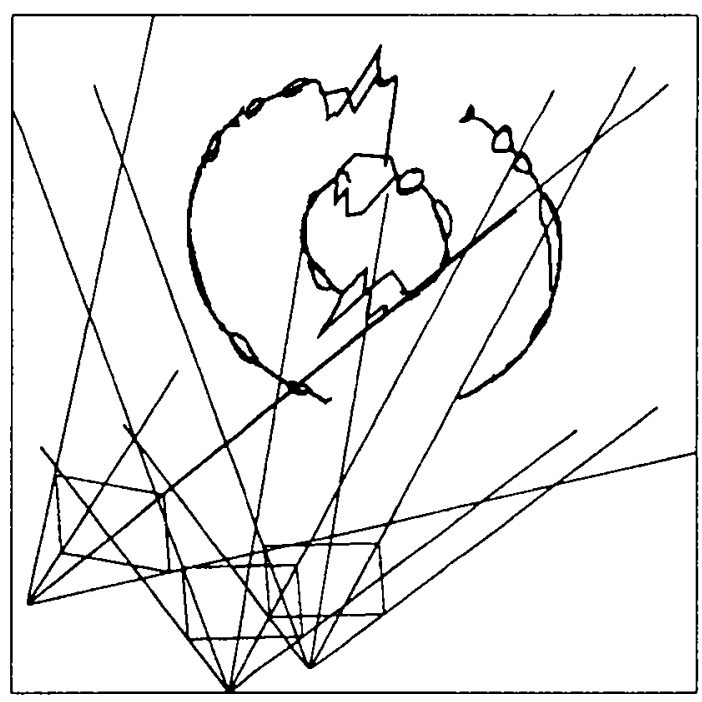

Figure 28: The reconstructed torus 


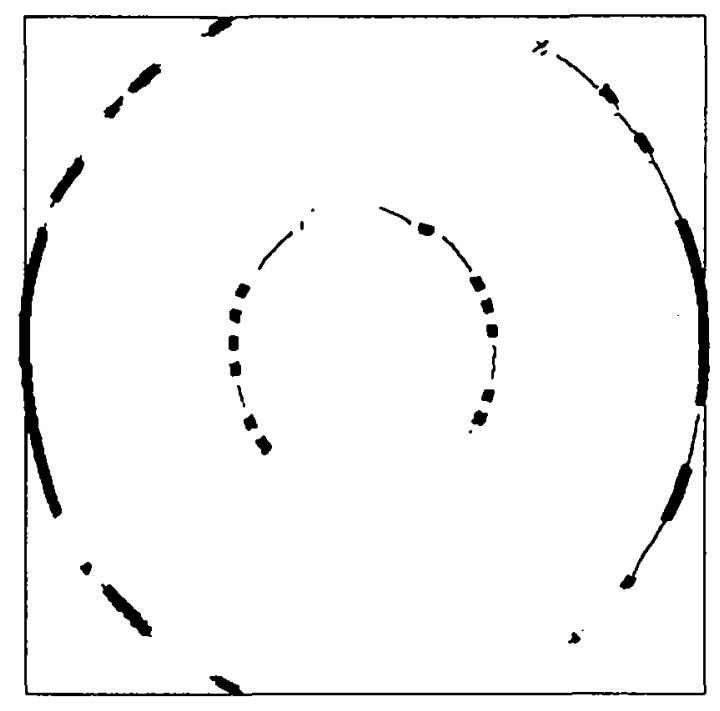

Figure 29: 'The sign of the ganssian curvature for the torus

There is still a point to be noted: we do not inprove the results by filtrering the produced whes. We have shown the direct output of the algorithm. We think that the quality of the results can be greatly improved if some smoothing techniques are applicil. We choose to not work in this direction as they will be part of a more sophisticated algorithm whose goal is to build a model of the object by fusing the information provided by a set of observations of the object. This set will be organized such that it covers a discrete sphere of the possible viewpoints.

\section{Conclusion}

In this article, we have shown that occluding edges are a rolust source of $3 \mathrm{D}$ information. Points on the rim can be accurately reconstructed and good estimates for the sccond order differential properties of the surface in the vicinity of the rim can be reliably computed. Nore work needs to be done to include this lind of processing in the framework of all active exploration of object shape. We are presently testing our algorithm on a large number of images representing screral different shapes of occluding edges. We also want to use the algorithm with more than three views. The supplementary views will be obtained by moving the object with a known motion. We think that this will improve accuracy greatly.

There is still another interesting point: is it possible 10 compute the same guantities for llic points of the rim of a surface observed by a camera with an unkiown motion. Faugeras [Fau90] proves that it is possible to recover the scene and the motion of the camera. if the observation is formed by a rigid curve. Infortunately the rim of a surface is not a rigid curve and wo camnot directly apply this result.

\section{A Proof of the relation between $r_{l,}, r_{i}$ and $r_{r}$}

We demonstrate this result by using the thcoren of Jocal inversion. 


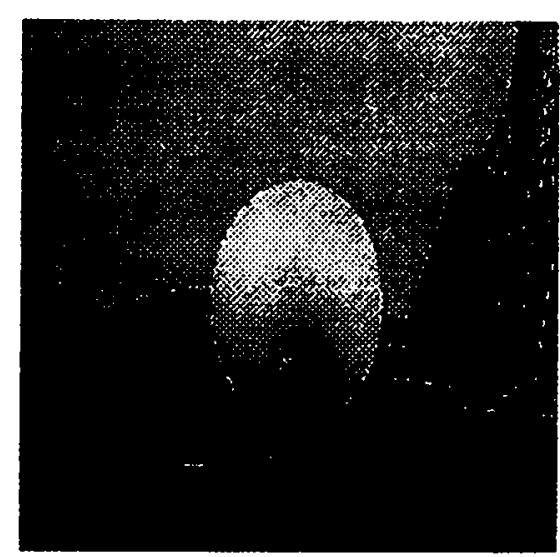

Camera 1

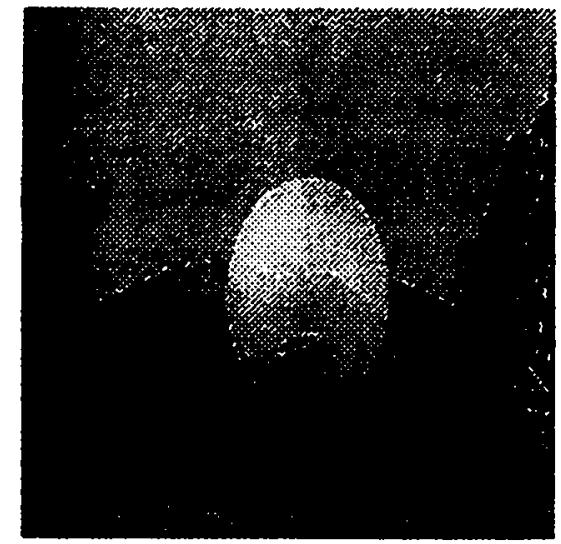

Camera 2

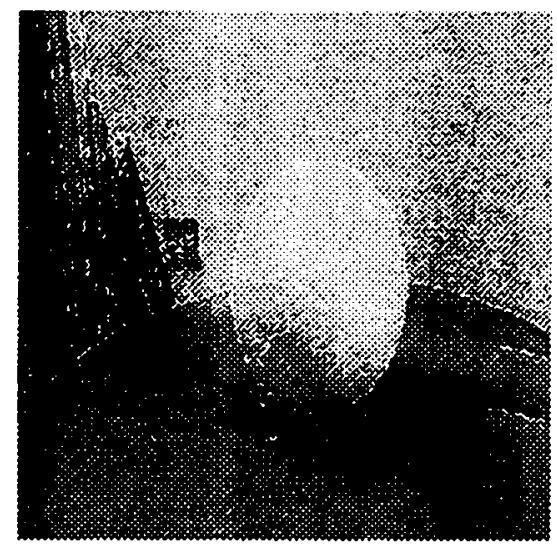

Camera 3

Figmre 30: Images of the triplet "ball" and the polygonal approximation 


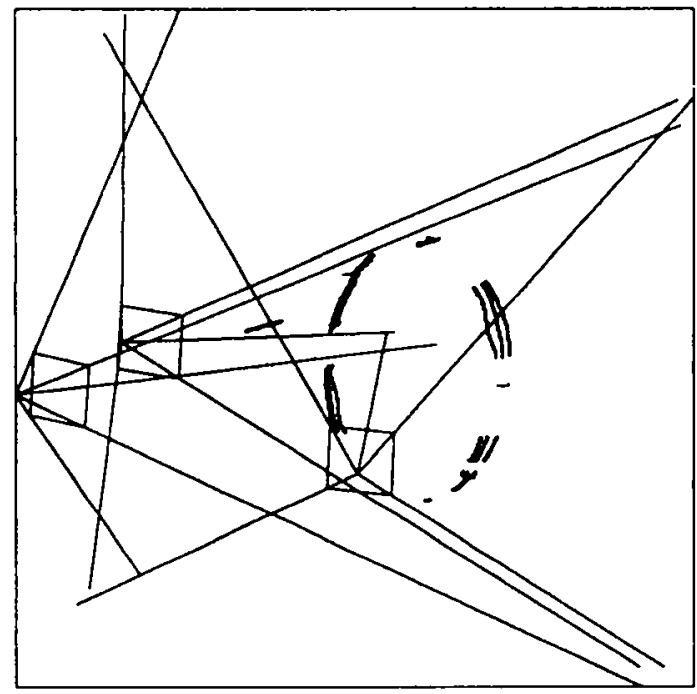

Figure 31: Reconstruction triplet "ball"

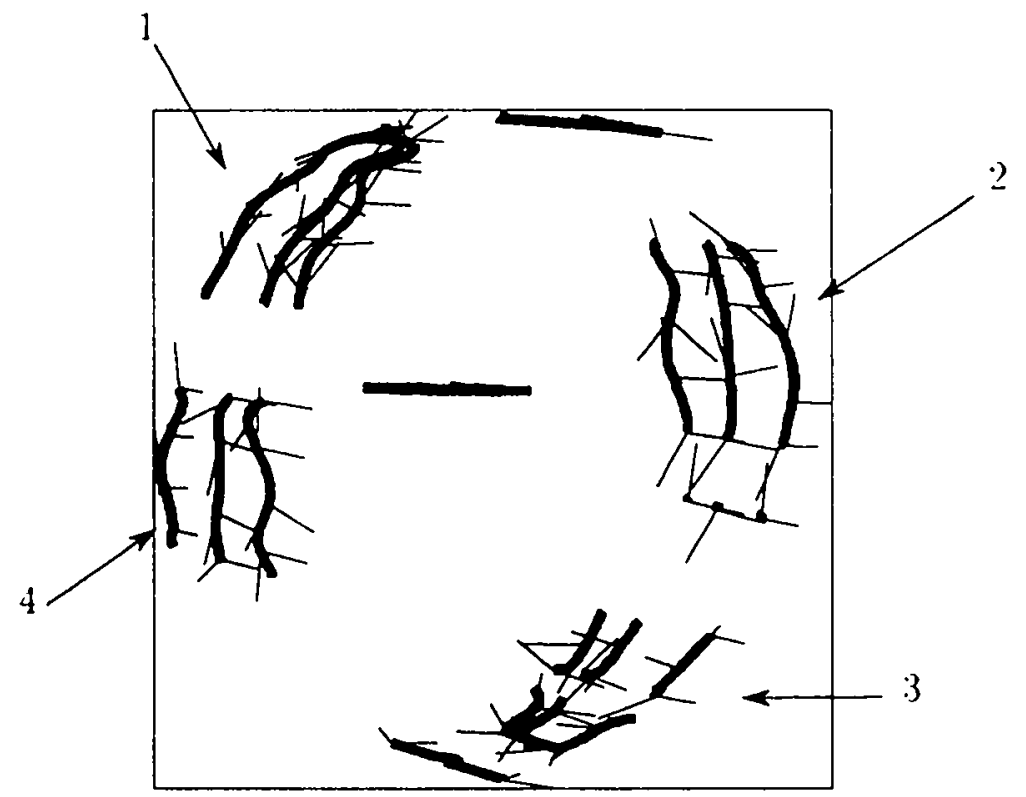

Figure 32: Reconst.ruction and principal direction triples "ball" 


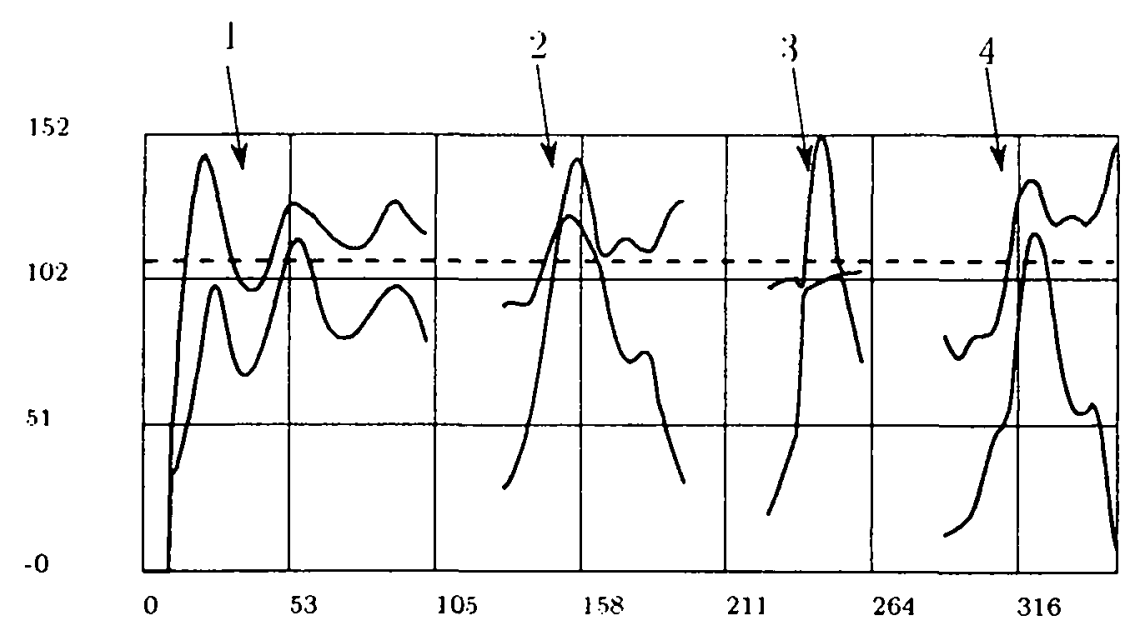

Figure 33: The conıuted radii of curvature (triplet "ball")

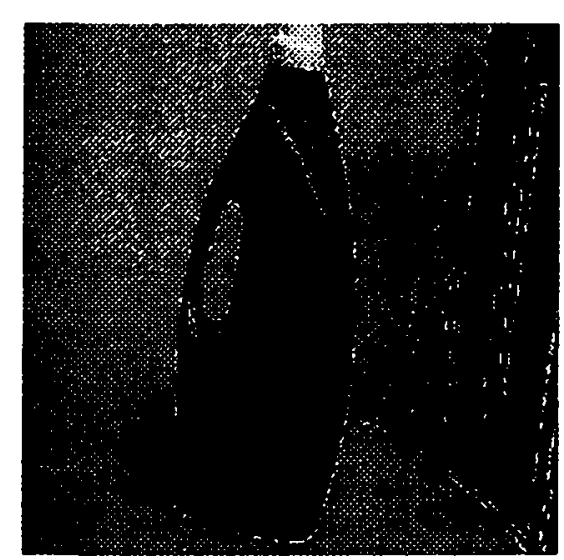

Camera 1

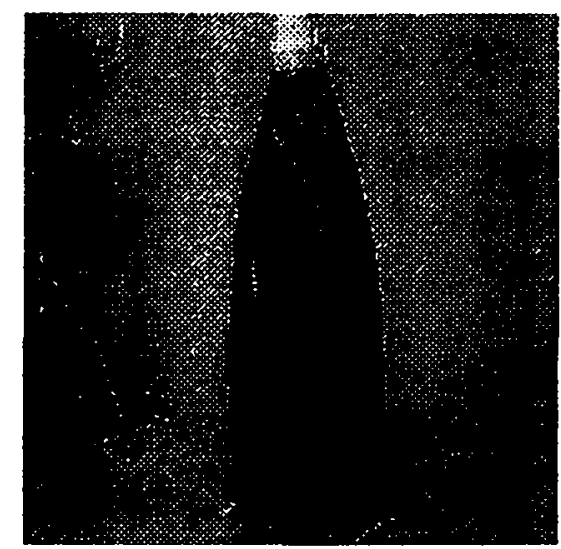

Camera 2

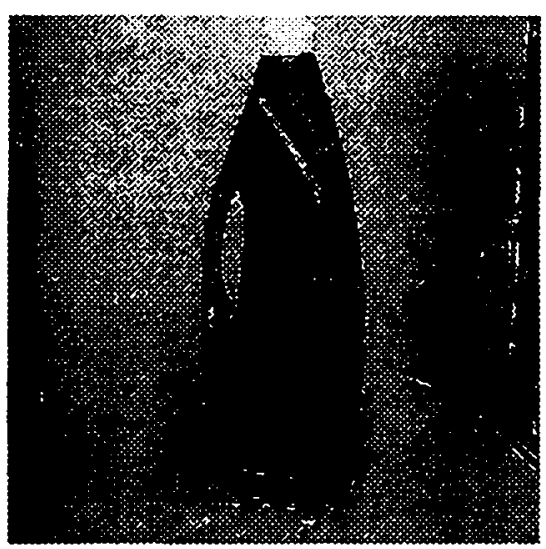

Cancra 3

Figure 34: lmages of the triplet "ho11l," and the polygonal approximations 


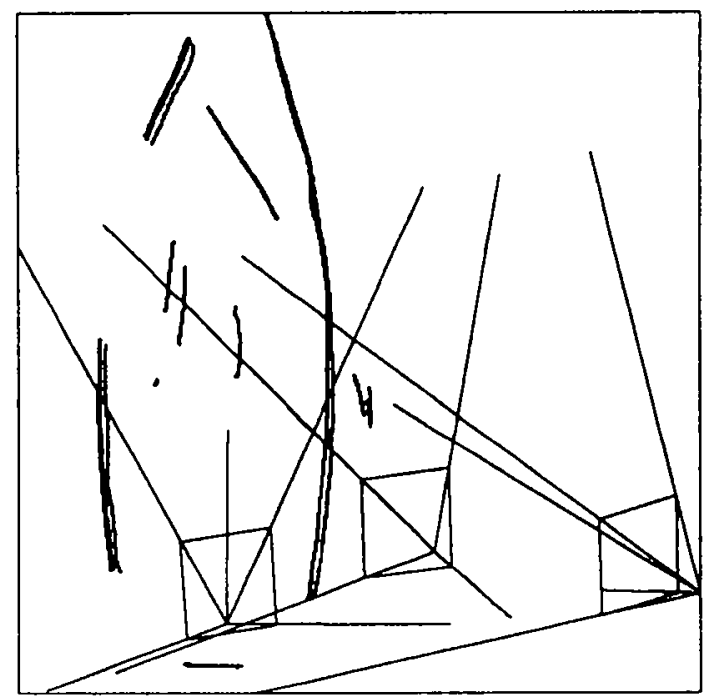

Figure 35: Reconstruction triplet "bottle"

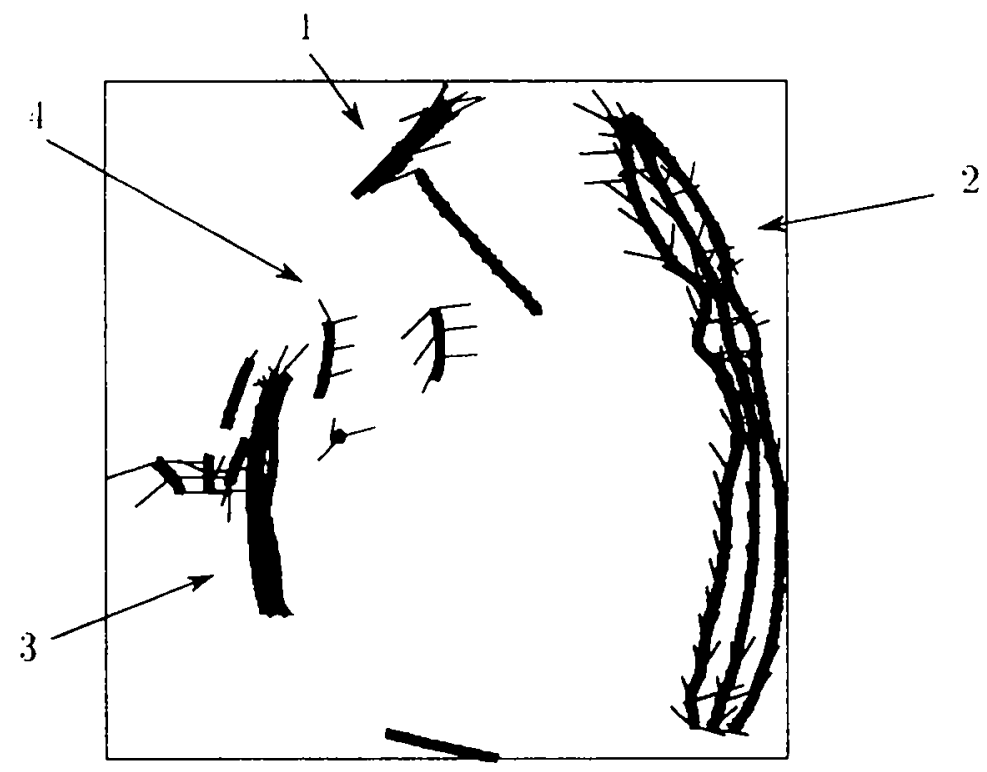

Figure 36: Reconstruction and principal directions (triplet "bot tle") 


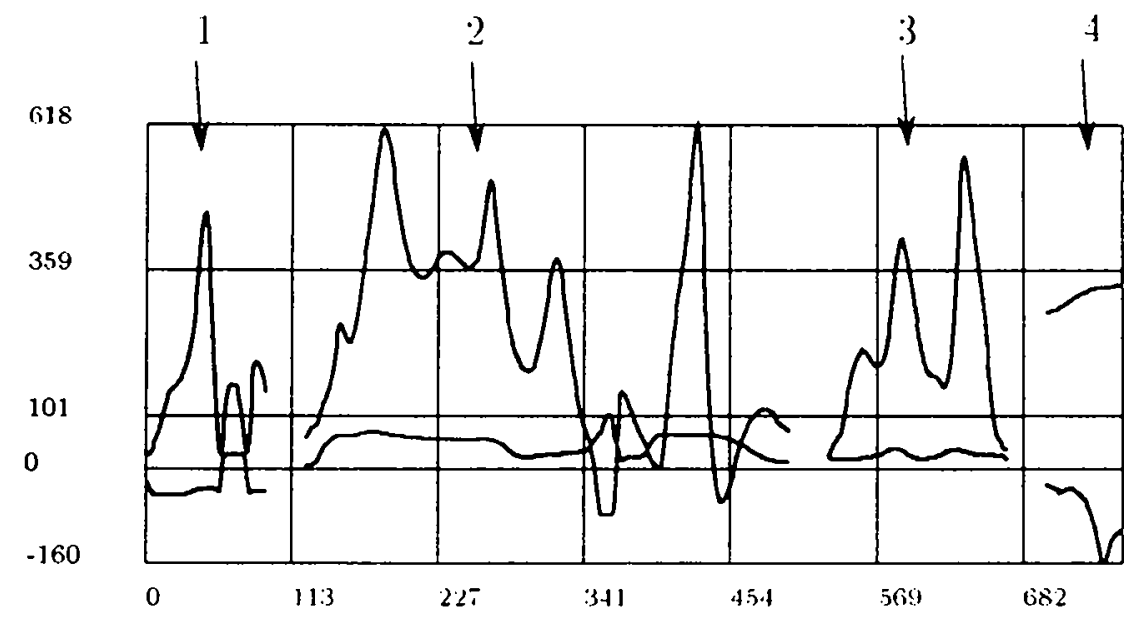

Figure 37: The computed radii of currature (triplet "bottle")

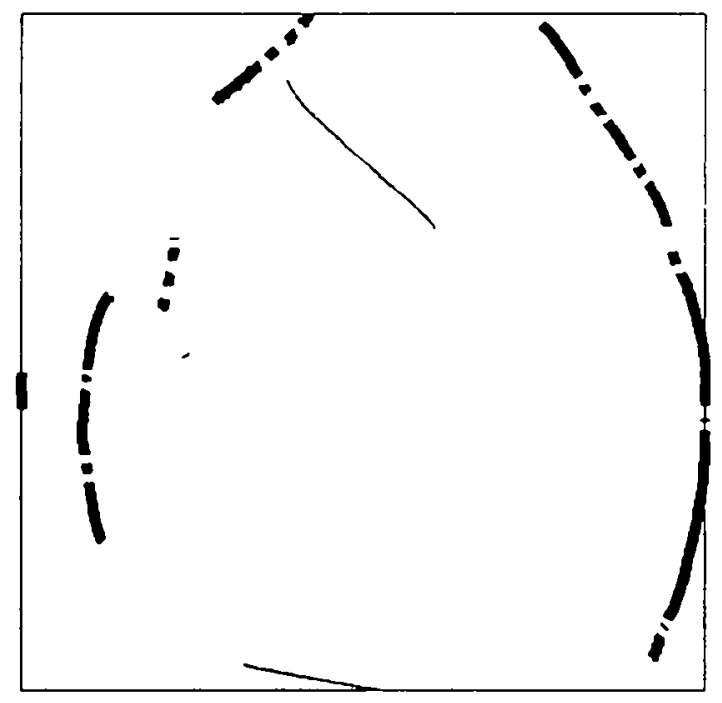

Figure 38: The sign of the ganssian curvature (triplet "bottle") 
Let $X=C^{\prime} P /\|C: P\|$ and $Z=N_{E}$ and $Y^{\prime}=Z \wedge X$. These 3 vectors form a france $\Lambda$ parametric equation of the surface $(\Sigma)$ in this frame is $M=\{x+\lambda, y, z(x, y)\}$ with $z(0,0)=$

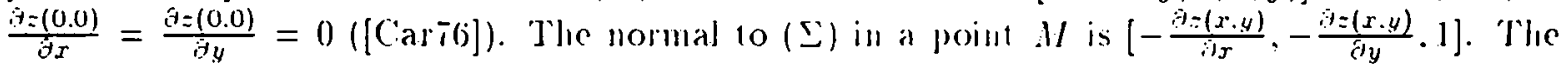
equation of the extremal boundary is

$$
N_{\Sigma} \cdot M=0 \Longrightarrow-(x+\lambda) \frac{\partial z(x, y)}{\partial x}-y \frac{\partial z(x, y)}{\partial y}+z(x, y)=0
$$

The equation (16) is an implicit equation with respect to the two variables $x$ and $y$. We write it:

$$
h(x, y)=-(x+\lambda) \frac{\partial z(x, y)}{\partial x}-y \frac{\partial z(x, y)}{\partial y}+z(x, y)=0
$$

We can solve this as $x=f(y)$ in the neighbourhood of $x=0, y=0$ if $\frac{\partial h(x . y)}{\partial x} \neq 0$ (local inversion theorem ).

First, we suppose $\frac{\partial h(0,0)}{\partial x}=-\lambda \frac{\hat{\theta}^{2} z(0,0)}{\partial x^{2}}=\frac{\partial^{2} \Sigma}{\partial x^{2}} \neq 0$.

Using the local inversion theorem, we get the derivatives of $x$ with respect to $y$ :

$$
\begin{gathered}
\frac{d x}{d y}=f^{\prime}(y)=-\frac{\partial h(x \cdot y)}{\partial y} / \frac{\partial h(x, y)}{\partial x} \\
\frac{d^{2} x}{d y^{2}}=f^{\prime \prime}(y)=-\left(\left(f^{\prime}(y)^{2} \frac{\partial^{2} h(x \cdot y)}{\partial x^{2}}+2 f^{\prime}(y) \frac{\partial^{2} h(x, y)}{\partial x \partial y}+\frac{\partial^{2} h(x, y)}{\partial y^{2}}\right)\right) / \frac{\partial h(x, y)}{\partial x}
\end{gathered}
$$

For $x=0$ and $y=0$. the partial deritatives of $/ 1$ are:

$$
\begin{gathered}
\frac{\partial h}{\partial x}=-\lambda \frac{\partial^{2} z}{\partial x^{2}} \quad \frac{\partial h}{\partial y}=-\lambda \frac{\partial^{2} z}{\partial x \partial y} \\
\frac{\partial^{2} h}{\partial x^{2}}=-\frac{\partial^{2} z}{\partial x^{2}}-\lambda \frac{\partial^{3} z}{\partial x^{3}} \frac{\partial^{2} h}{\partial y^{2}}=-\frac{\partial^{2} z}{\partial y^{2}}-\lambda \frac{\partial^{3} z}{\partial y^{3}} \frac{\partial^{2} h}{\partial x \partial y}=-\frac{\partial^{2} z}{\partial x \partial y}-\lambda \frac{\partial^{3} z}{\partial x^{2} y}
\end{gathered}
$$

The extremal boundary is the curve:

$$
P(y)=\left[\begin{array}{c}
f(y)+\lambda \\
y \\
z(f(y) \cdot y)
\end{array}\right]
$$

The first and second partial derinatives are:

$$
\begin{gathered}
P^{\prime}(y)=\frac{d P(y)}{d y}=\left[\begin{array}{c}
f^{\prime}(y) \\
1 \\
f^{\prime}\left(y \frac{\partial z z(x, y)}{\partial x}+\frac{\partial z(x, y)}{\partial y}\right.
\end{array}\right] \\
P^{\prime \prime}(y)=\frac{d^{2} P(y)}{d y^{2}}=\left[\begin{array}{c}
f^{\prime \prime}(y) \\
0 \\
f^{\prime}(y)^{2} \frac{\partial^{2} z(x, y)}{\partial x^{2}}+2 f^{\prime}(y) \frac{\partial^{2} z(x, y)}{\partial x \partial^{\prime} y}+\frac{\partial^{2} z(x, y)}{\partial y^{2}}
\end{array}\right]
\end{gathered}
$$

For $x=0$ and $y=0$ : 


$$
P(0)=\left[\begin{array}{l}
\lambda \\
0 \\
0
\end{array}\right] \quad P^{\prime}(0)=\left[\begin{array}{c}
f^{\prime}(0) \\
1 \\
0
\end{array}\right] \quad I^{\prime \prime}(0)=\left[\begin{array}{c}
f^{\prime \prime}(0) \\
0 \\
f^{\prime}(0)^{2} \frac{\partial^{2} z}{\partial r^{2}}+2 f^{\prime}(y) \frac{\partial^{2} z}{\partial \partial^{2} \partial y}+\frac{\partial^{2} z}{\partial y^{2}}
\end{array}\right]
$$

In order to do analyze the properties of $(r)$. we have to define more precisely the camera and to introduce a frame $[U, \mathrm{~V}, W]$. W is the optical axis. $l$ and $F$ are a basis of the image plane. The only constraint on $l^{\prime}$ is $l^{\prime} \cdot W=0$, so we call add the constraint $l^{\prime} \cdot S^{\prime}=0$ (we choose $l$ ? in the plane $X-Z$ ) (figure 39 ).

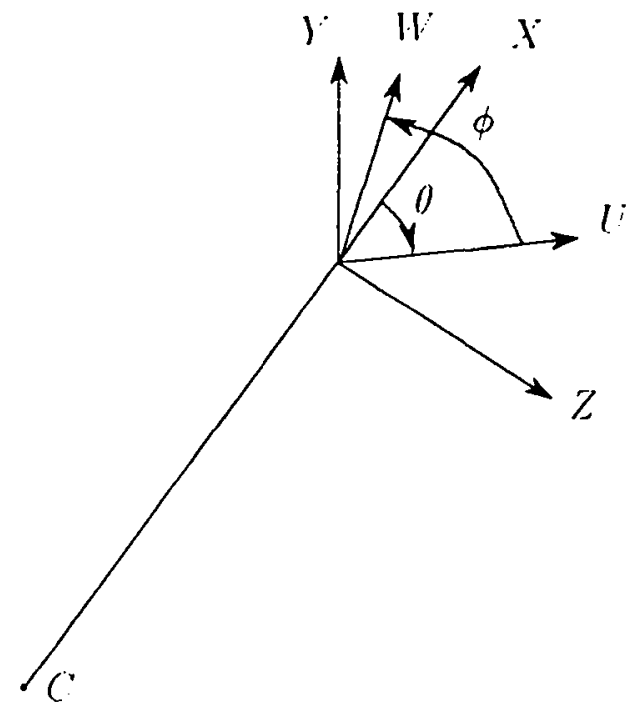

Figure 39: The wo frames

$$
l=[\cos (\theta) \cdot 0 \cdot \sin (\theta)]^{7}
$$

IT is orthogonal to $l$. If is defined by choosing the angle between 15 and one of the vector $[X . Y, Z]$. We take:

$$
\begin{gathered}
H=[-\sin (\theta) \cos (\phi) \cdot \sin (\phi) \cdot \cos (\theta) \cos (\phi)]^{T} \\
\forall=H \wedge U=[\sin (\phi) \sin (\theta), \cos (\phi),-\sin (\phi) \cos (\theta)]^{T}
\end{gathered}
$$

The point $\nu(y)$ which is the image of $P(y)$ is defined by:

$$
\mu(y)=\left[\begin{array}{l}
\frac{U \cdot P(y)}{W \cdot P(y)} \\
\frac{V \cdot P(y)}{\| \cdot P(! !)}
\end{array}\right]
$$

We compute the first and second derivatives of $p(y)$ :

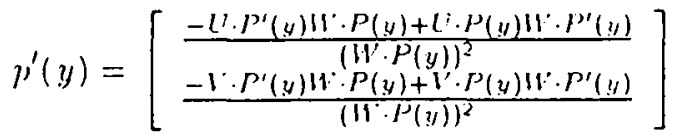




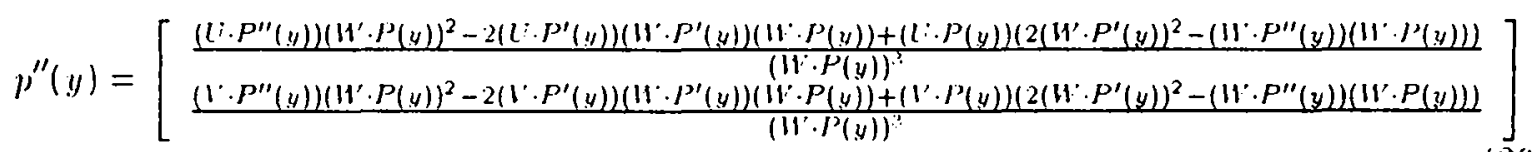

If we simplify these expressions. we get:

$$
\mu^{\prime}(y)=\left[\begin{array}{c}
-\frac{\sin (\phi) \cos (\theta)}{\lambda \cos (\phi)^{2} \sin (\theta)^{2}} \\
-\frac{1}{\lambda \sin (\theta) \cos (\phi)^{2}}
\end{array}\right]
$$

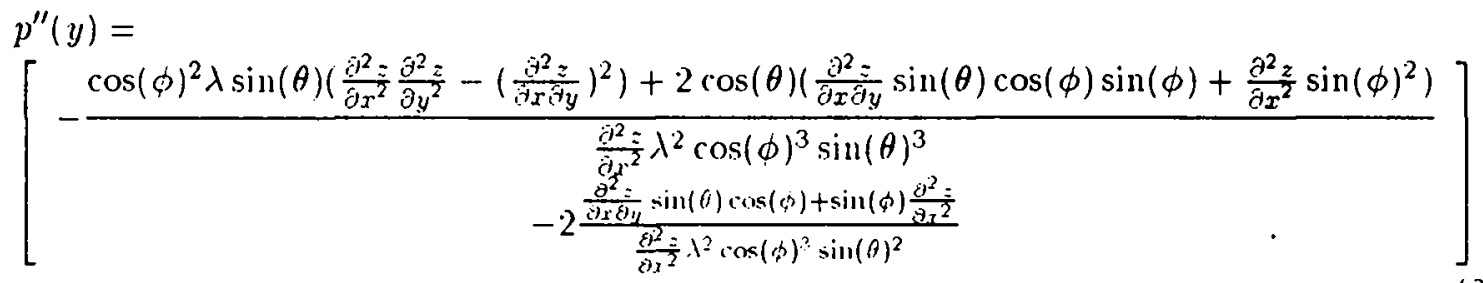

With respert to the derivatives of the coordinates of $p(y)$. the expression of $k_{t}$ is:

$$
r_{i}=\frac{p^{\prime}(y)_{u} p^{\prime \prime}(y)_{1}-p^{\prime \prime}(y)_{u} p^{\prime}(y)_{1}}{\left(p^{\prime}(y)_{u}^{2}+p^{\prime}(y)_{l^{\prime}}^{2}\right)^{(3 / 2)}}
$$

So we have:

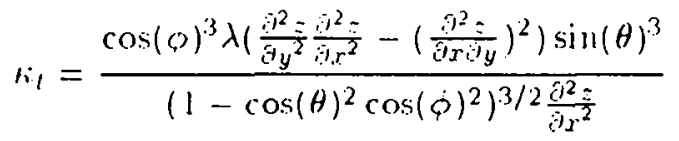

In the same way. We can compute the radial curvatuse: the radial curve is the intersection of the plane $X-Z$ with ( $(\Sigma)$. Consecuenty its equation is $M=[z(x, 0) . x]$ in the basis $(Z, X)$ of the plane $x-\%$. After computation of the deritatives. we obtain:

$$
\kappa_{r}=\frac{\partial^{2} z}{\partial x^{2}}
$$

The gaussian curvalure for a point on a surface represented as a Monge patch is:

$$
r_{y}=\frac{\frac{\partial^{2} z(x \cdot y)}{\partial x^{2}} \frac{\partial^{2} z(r \cdot y)}{\partial y^{2}}-\left(\frac{\partial^{2} z(x \cdot y)}{\partial x y^{2} y}\right)^{2}}{\left(1+\left(\frac{\partial z(r \cdot y)}{\partial x}\right)^{2}+\left(\frac{\partial z(x \cdot y)}{\partial y y}\right)^{2}\right)^{2}}
$$

For $x=0$ and $y=0$. il simplifies in:

$$
\kappa_{i g}=\frac{\partial^{2} z}{\partial x^{2}} \frac{\partial^{2} z}{\partial y^{2}}-\left(\frac{\partial^{2} z}{\partial x \partial y}\right)^{2}
$$

By taking into accomnt the equations: (30). (31) and (3:3) we get the simple relation:

$$
r_{i g}=\frac{\left(1-\cos (\theta)^{2} \cos (\phi)^{2}\right)^{3 / 2}}{\cos (\phi)^{3} \sin (\theta)^{3}} \frac{r_{i}}{\lambda}
$$


The denominator of this couation cannol be zero because $X$ and $W$ cannot be orthogonal as $\mathbb{W}$ is the optical axis and $X$ is an optical ray.

$\theta$ and $\phi$ can be determined from $X$. \% and $H$ or expitalently from $C P . N_{\Sigma}$ and $H^{\prime}$ as $X=C P /\|C P\|$ and $Z=N_{\text {I }}$

$$
\sin (\phi)=W \cdot(\% \wedge x) \operatorname{and} \cos (\theta)=\left(W^{\prime} \cdot Z\right) / \cos (\phi)
$$

so we can write:

$$
n\left(W .\left(P . N_{\Sigma}\right)=\frac{\left(1-\cos (\theta)^{2} \cos (\phi)^{2}\right)^{3 / 2}}{\cos (\phi)^{3} \sin (\theta)^{3}}\right.
$$

The equations (34) and (35) vield the following result:

$$
\kappa_{g}=a\left(H, C: P . \Lambda_{\Sigma}\right) \frac{\kappa_{t} \kappa_{r}}{\lambda}
$$

The equation (31) proves that the condition $\frac{\partial h(x, y)}{d x} \neq 0$ is equivalent to $k_{r} \neq 0$.

If $\frac{i h(x, y)}{\partial x}=0$ and $\kappa_{g} \neq 0$, equation (33) implies that $\frac{\partial h(x, y)}{\partial y} \neq 0$. We apply the local inversion theorem to the equation (16) and solve it as $y=g(x)$. We obtain immediately that the tangent vector to the rim $(r)$ is equal to zero at $p: p$ is a singular point.

Remark 1 We have built during this proof an analytical representation of the extremal boundary $(R)$ which can be used to compute some of the properties of the rim $(R)$. The curvature $t$ and the torsion $\theta$ have very complex expressions. The most interesting point is that the normal curvature $k_{n}$ of $(R)$ is proportional to the gaussian curvature:

$$
\kappa_{n}=\frac{\partial^{2} z}{\partial x^{2}}\left(\frac{\partial^{2} z}{\partial y^{2}} \frac{\partial^{2} z}{\partial x^{2}}-\left(\frac{\partial^{2} z}{\partial x \partial y}\right)^{2}\right) /\left(\left(\frac{\partial^{2} z}{\partial x \partial y}\right)^{2}+\left(\frac{\partial^{2} z}{\partial x^{2}}\right)^{2}\right)=i_{r} \kappa_{y}\left(\left(\frac{\partial^{2} z}{\partial x \partial y}\right)^{2}+\left(\frac{\partial^{2} z}{\partial x^{2}}\right)^{2}\right)
$$

Remark 2 Using the same principle. we cau prove similar relations for other models of cameras:

In the orthographic casc. we obtain the relation which has been demonstrated by hoenderinli [lioes-1]:

$$
r_{i g}=n_{t} k_{i}
$$

In the spherical case. we obtain the relation which has been derived by Blake and ('ipolla [BC:90] using a different method:

$$
r_{y}=r i p_{i} / \lambda
$$

$r_{p}$ is the geodesic curvature of $(r)$ as a curve on the wnit splere.

\section{B Computation of the second fundamental form}

We consider a point $P$ on the surface. We note $X=[x, y, z]^{T}$. its coordinates. We consider the plane tangent to the surface at $P$. This plane is defined by its vector normal $n$. and its distance $p$ to the origin. Its equation can be written:

$$
n . x-\mu=0
$$

$n$ can be represcuted as a function of its Euler angle: 


$$
\left\{\begin{array}{l}
n_{x}(\theta, \phi)=\cos (\phi) \cos (\theta) \\
n_{y}(\theta, \phi)=\cos (\phi) \sin (\theta) \\
n_{z}(\theta, \phi)=\sin (\phi)
\end{array}\right.
$$

We derive the equation $(-10)$ and we obtain the two following equations:

$$
\left\{\begin{aligned}
&-\cos (\phi) \sin (\theta) \cdot x+\cos (\phi) \cos (\theta) y-\frac{\partial p(\theta . \phi)}{\partial \theta}=0 \\
&-\sin (\phi) \cos (\theta) \cdot r-\sin (\phi) \sin (\theta) y+\cos (\phi) z-\frac{\partial p(\theta . \phi)}{\partial \phi}=0
\end{aligned}\right.
$$

We find the expression of $X=[x, y, z]^{T}$, by solving the following system of three lincar equation: (40), one equation: (-11), two equations.

We find:

$$
\left\{\begin{array}{l}
x=\cos (\phi) \cos (\theta) p(\theta, \phi)-\sin (\phi) \cos (\theta) \frac{\partial p(\theta, \phi)}{\partial \phi}-\frac{\sin (\theta)}{\cos (\phi)} \frac{\partial p(\theta, \phi)}{\partial \theta} \\
y=\sin (\theta) \cos (\phi) p(\theta, \phi)-\sin (\theta) \sin (\phi) \frac{\partial p(\theta, \phi)}{\partial \phi}+\frac{\cos (\theta)}{\cos (\phi)} \frac{\partial p(\theta, \phi}{\partial \phi} \\
z=\sin (\phi) p(\theta, \phi)+\cos (\phi) \frac{\partial p(\theta, \phi)}{\xi \theta}
\end{array}\right.
$$

From these expressions $(+2)$. we derive the expression of $\frac{\partial x(\theta, \phi)}{\partial \theta}$ and $\frac{\dot{\partial} x\left(\theta, \phi_{1}\right)}{\dot{\partial} \phi}$.

The first fundamental form is:

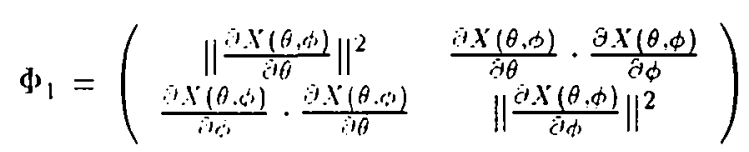

As uswal. we note:

$$
E=\left\|\frac{\partial X(\theta, \phi)}{\partial \theta}\right\|^{2} \quad F=\frac{\partial X(\theta, \phi)}{\partial \theta} \cdot \frac{\partial X(\theta . \phi)}{\partial \phi} \quad G=\left\|\frac{\partial X(\theta . \phi)}{\partial \phi}\right\|^{2}
$$

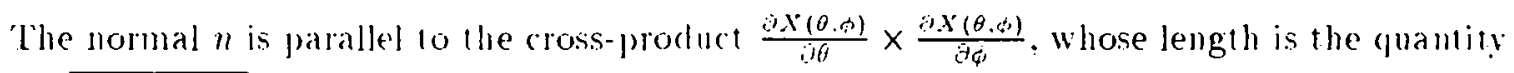
$H=\sqrt{\left(E G^{\prime}-F^{2}\right)}$.

From these quantities. We can derive the second fundamental quadratic form. It is expressed with the following alements:

$$
L=\frac{\partial^{2} X(\theta, \phi)}{\partial \theta^{2}} \cdot n(\theta . \phi) \quad M=\frac{\partial^{2} X(\theta, \phi)}{\partial \theta \partial \phi} \cdot n(\theta, \phi) \quad N=\frac{\partial^{2} X(\theta, \phi)}{\partial \phi)^{2}} \cdot n(\theta, \phi)
$$

Note that the expression of $\mathcal{X}(\theta, \phi)$ involves derivatives of the first order. As a consequence

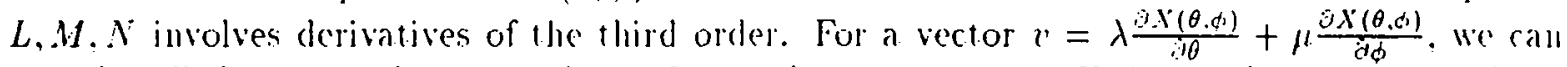
consider all the curves drawn on the surface and langemt to $v$ at $X(\theta . \phi)$. These curves all have the same normal curvature. the ratio $\frac{\Phi_{2}(u)}{\Phi_{1}\left({ }^{\prime \prime}\right)}$.

$\Phi_{2}$ defines a linear mapping $\phi$ from the tangent plane of the surface at $\mathcal{X}(\theta, \phi)$ to the same plane. $\psi$ is defined such that $\Phi_{2}(v)=\psi(v) \cdot v$. The principal directions and the principal curvatures are the eigenvectors and the eigenvalues of $\psi$. The coordinates of the principal directions are obtained as the solution of the following equation:

$$
(F L-E M) \lambda^{2}+(G l-E N) \lambda \mu+(G M-F N) \mu^{2}=0
$$

These coordinates are express in the frame $\left(\frac{a x(0,6)}{\theta 0} \cdot \frac{x(\theta, 0)}{i \phi}\right)$. 
The rigemwlues are obtained as the solution of the fullowing equation:

$$
\left(L C i-F^{2}\right) \rho^{2}-\left(L(i+E N-2 F M) \rho+\left(L N-M^{2}\right)=0\right.
$$

These parameters are inmariants of the surface and in particular they do not depend on the choice of the parametrization. It is possible to derive all these computations with the liclp of a symbolic computation package and this is how we obtain the curvatures. The expressioms that we obtained do not depend on the third order derivatives of $p(\theta, \phi)$.

Now we give the expression of the different guantities involved in the computations of the principal curvatures and principal directions.

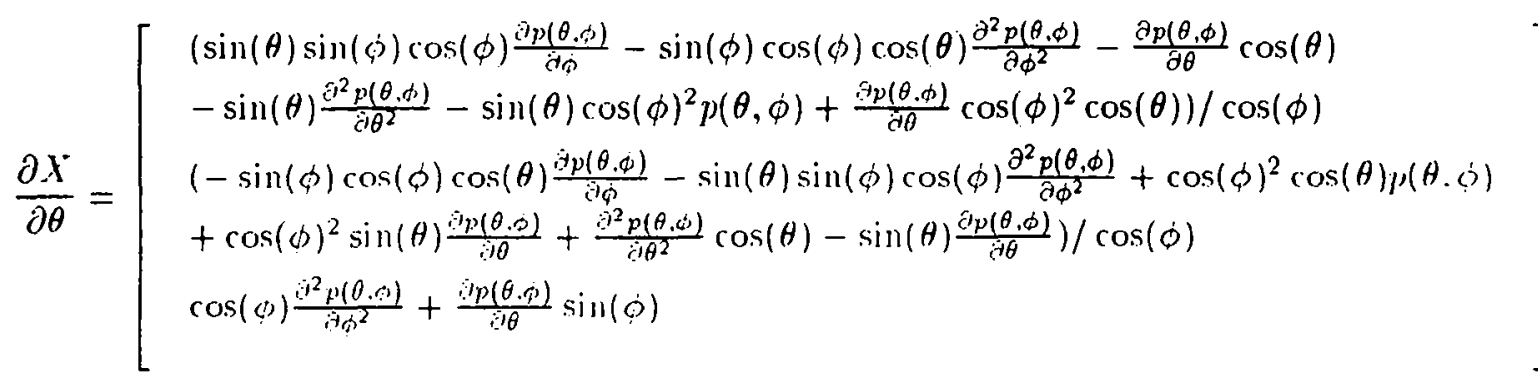

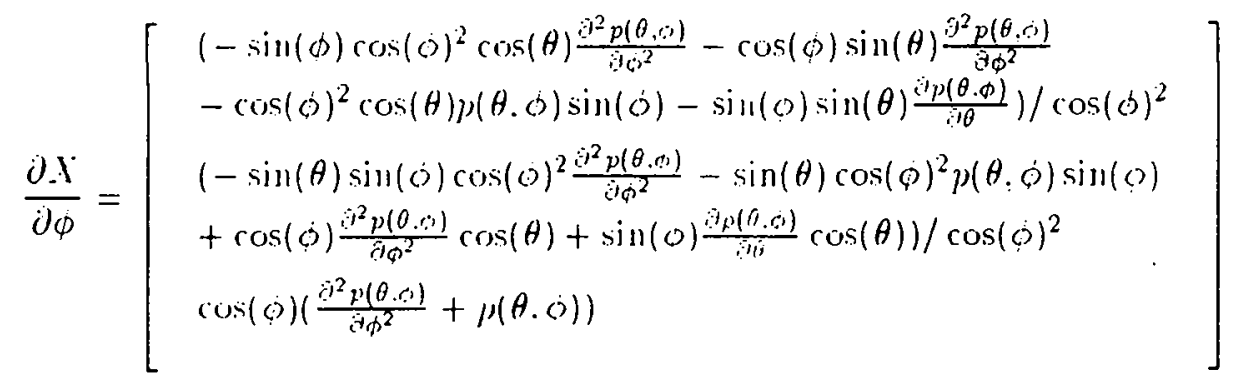

The following step is the second order derivatives of $\mathcal{X}(\theta, \delta)$ :

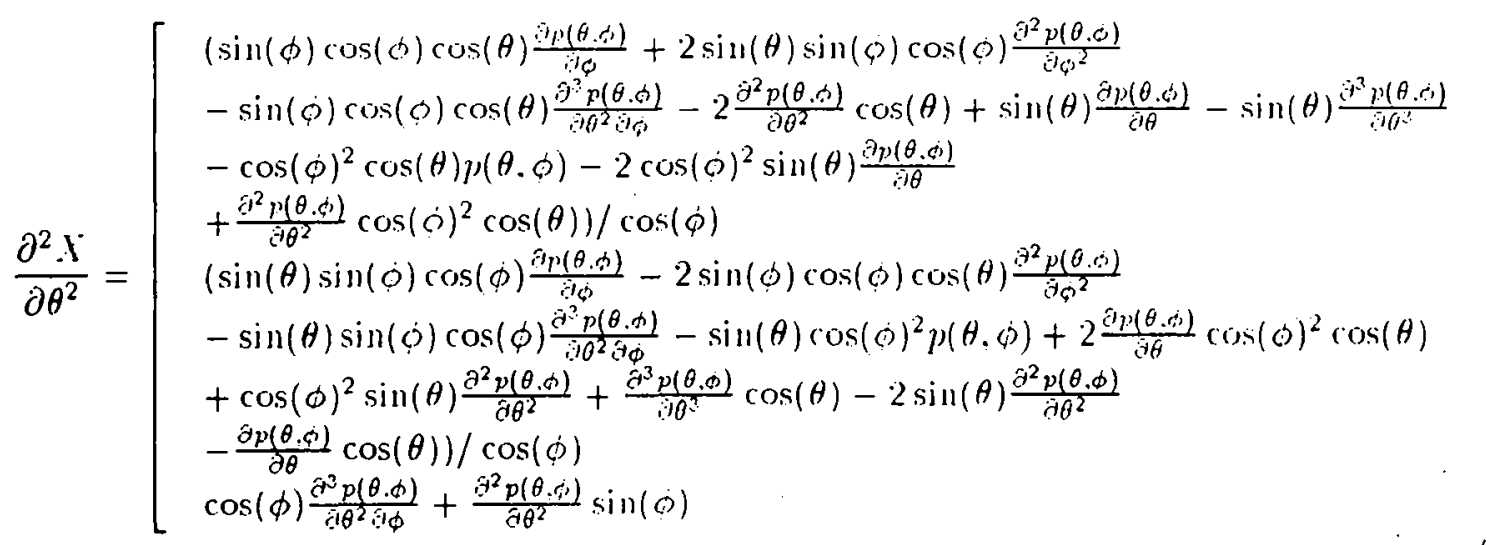




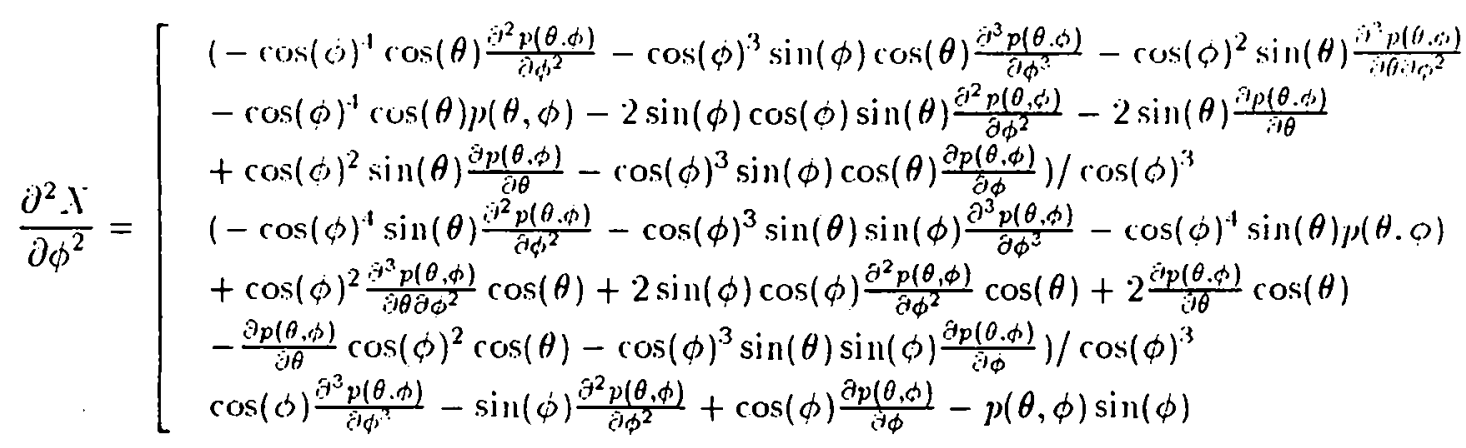

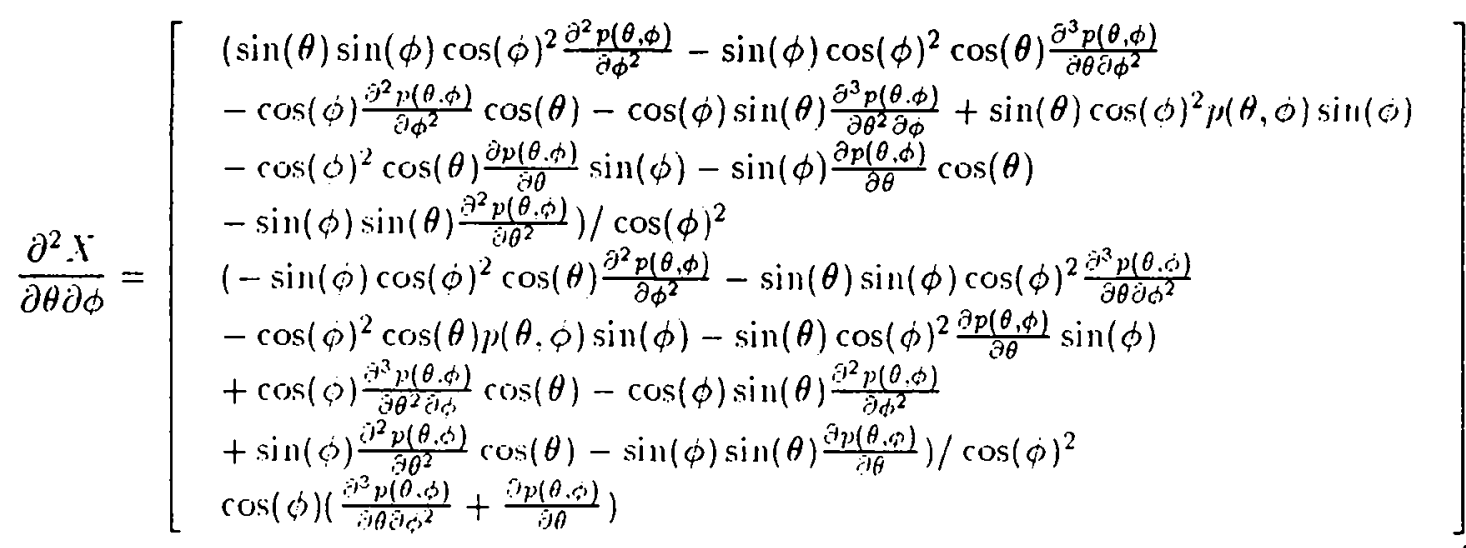

The cuefficients of the first guadratic form are:

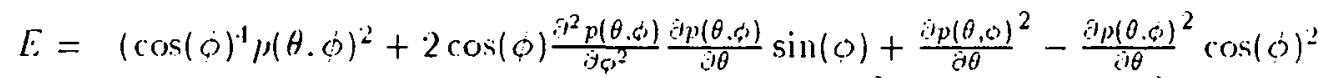

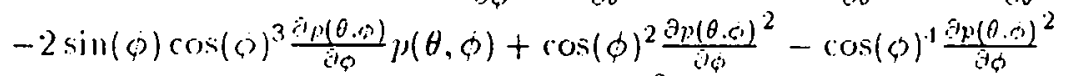

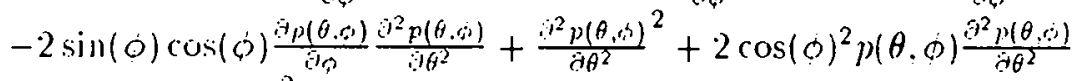

$$
\begin{aligned}
& \left.+\cos (\phi)^{2} \frac{i^{2} p(\theta, \phi)^{2}}{\partial^{2} \phi^{2}}\right) / \cos (\phi)^{2}
\end{aligned}
$$

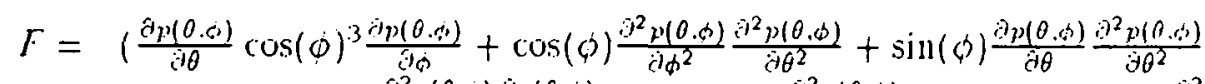

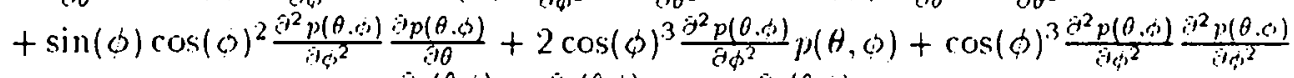

$$
\begin{aligned}
& +2 \cos (\phi)^{2} p(\theta \cdot \phi) \sin (\phi) \frac{\partial p\left(\theta, \sigma_{0}\right)}{\partial \theta}-\frac{i p(\theta, \phi)}{c^{\prime} \theta} \cos (\phi) \frac{\partial p(\theta, \phi)}{\partial \phi} \\
& \left.-\cos (\phi)^{2} \frac{\partial^{2} p\left(\theta, c^{i}\right)}{\dot{a}^{2} \phi^{2}} \sin (\phi) \frac{\partial p(\theta . \phi)}{\dot{\theta} \phi \phi}\right) / \cos (\phi)^{3} \\
& G=\left(2 \cos (\phi)^{4} \frac{\theta^{2} p(\theta, \phi)}{\partial \phi^{2}} p(\theta, \phi)+\cos (\phi)^{4} p(\theta, \phi)^{2}+\cos (\phi)^{4} \frac{\hat{\theta}^{2} p(\theta, \phi)^{2}}{\partial \phi^{2}}-\frac{\dot{\partial} p(\theta \cdot(\dot{)})}{\partial \theta} \cos (\phi)^{2}+\right. \\
& \left.\frac{\hat{\partial} p(\theta, \phi))^{2}}{\partial \theta}+2 \cos (\phi) \frac{\partial^{2} p(\theta, \phi)}{\partial \phi^{2}} \sin (\phi) \frac{\hat{\partial} p(\theta, \phi)}{\partial \theta}+\cos (\phi)^{2} \frac{\partial^{2} p(\theta, \phi)^{2}}{\partial \theta \phi^{2}}\right) / \cos (\phi)^{4}
\end{aligned}
$$

The coefficients of the second gundratic form are: 


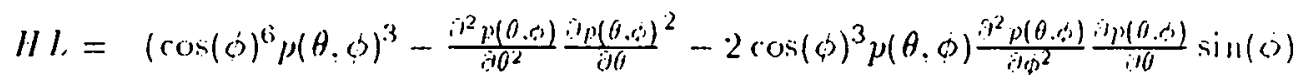

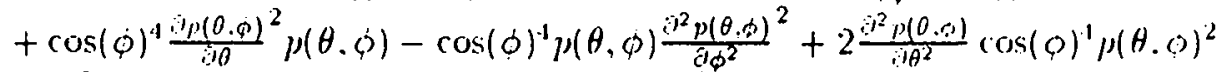

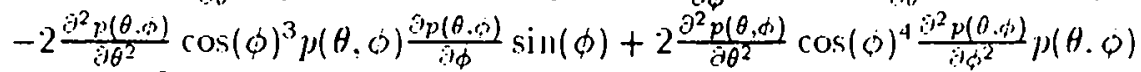

$$
\begin{aligned}
& +{\frac{\partial^{2} p(\theta .,)^{2}}{\dot{c}^{\prime} \theta^{2}}}^{2} \cos (\phi)^{2} p(\theta, \phi)+\cos (\phi)^{6} p(\theta, \phi)^{2} \frac{\partial^{2} p(\theta, \phi)}{\partial \phi^{2}} \\
& -2 \sin (\phi) \cos (\phi)^{5} \frac{\partial p(\theta, \phi)}{\dot{i}^{\prime} \phi} \frac{\varepsilon^{2} p(\theta, \phi)}{\partial \phi^{2}} p(\theta, \phi)-2 \sin (\phi) \cos (\phi)^{5} \frac{\dot{\partial} p(\theta, \phi)}{\dot{\partial} \phi \phi} \mu(\theta, \phi)^{2}
\end{aligned}
$$

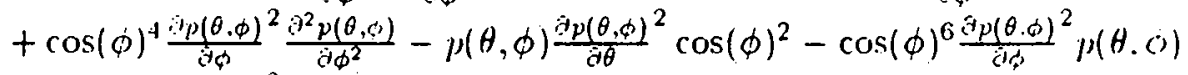

$$
\begin{aligned}
& -\cos (\phi)^{6} \frac{\dot{\partial} p(\theta, \phi)^{2}}{\partial \phi} \frac{\partial^{2} p(\theta, \phi)}{\partial \phi^{2}}-2 \sin (\phi) \cos (\phi)^{3} \frac{\partial p(\theta, \phi)}{\partial \dot{\phi}} \frac{\partial^{2} p(\theta, \phi)}{\partial \phi^{2}} \frac{\partial^{2} p(\theta, \phi)}{\partial \theta^{2}} \\
& +\cos (\phi)^{4} \frac{\hat{\theta} p(\theta, \phi)}{\hat{\partial} \phi} p(\theta, \phi)+2 \cos (\phi)^{2} \frac{\partial p(\theta, \phi)}{\partial \phi} \frac{\hat{\sigma}^{2} p(\theta, \phi)}{\partial \phi^{2}} \frac{\partial p(\theta, \phi)}{\partial \theta} \\
& -2 \cos (\phi)^{4} \frac{\partial p(\theta, \phi)}{\partial \phi} \frac{\partial^{2} p(\theta, \phi)}{\partial \phi^{2}} \frac{\partial p\left(\theta, \phi_{1}\right)}{\partial \theta}-\cos (\phi)^{3} \frac{\partial p(\theta, \phi)}{\partial \phi} \frac{\partial p(\theta, \phi)^{2}}{\partial \theta} \sin (\phi) \\
& +\cos (\phi) \frac{\hat{s} p(\theta, \phi)}{\dot{\partial} \phi} \frac{\partial p(\theta, \phi))^{2}}{\partial \theta} \sin (\phi)+\sin (\phi) \cos (\phi)^{3} \frac{\partial p(\theta, \phi)}{\partial \phi}{\frac{\sigma^{2} p(\theta, \phi)}{\partial \theta \phi^{2}}}^{2}
\end{aligned}
$$

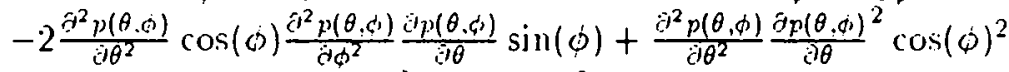

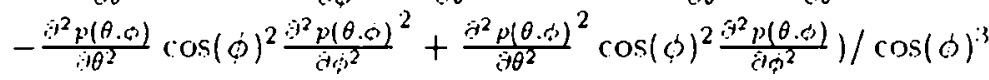

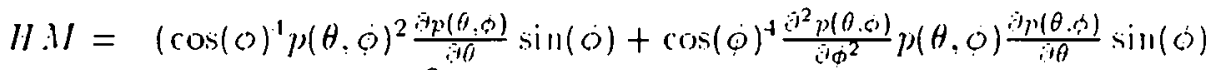

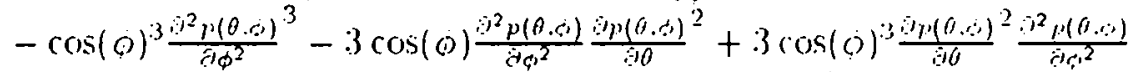

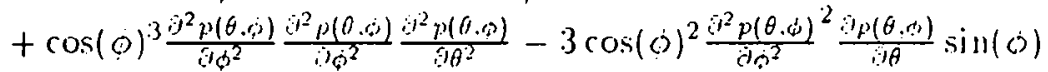

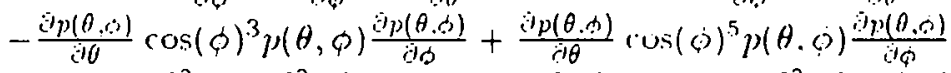

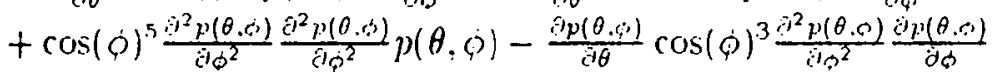

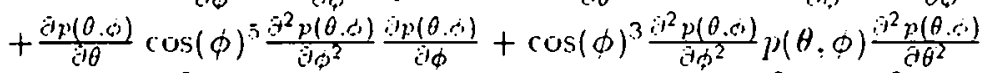

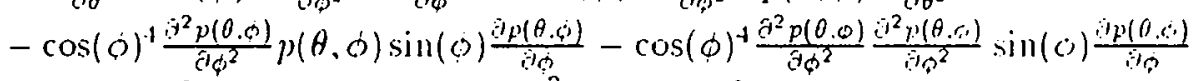

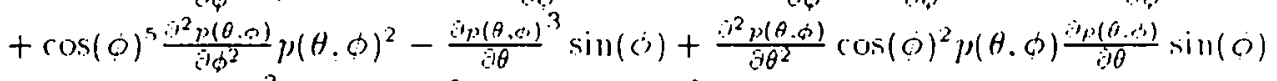

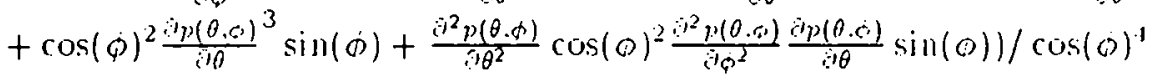

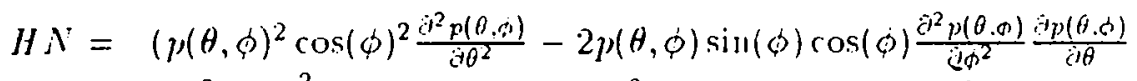

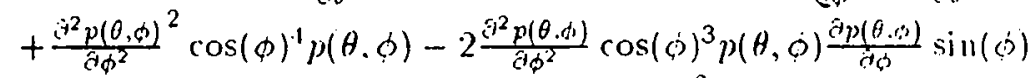

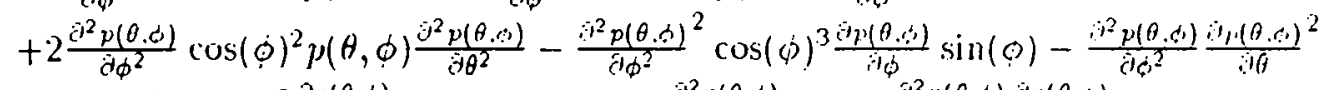

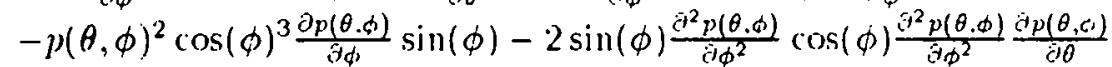

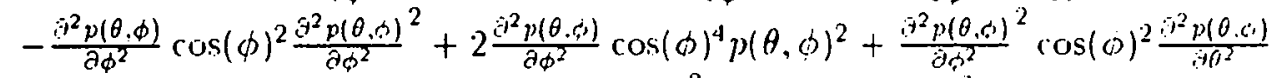

$$
\begin{aligned}
& +p(\theta, \phi)^{3} \cos (\phi)^{4}-p(\theta, \phi) \cos (\phi)^{2}{\frac{\partial^{2} p\left(\theta, \phi^{2}\right)^{2}}{\partial \phi^{2}}}^{2} p(\theta, \phi) \frac{\partial p(\theta, \phi)}{\partial \theta}^{2} \\
& \left.+p(\theta, \phi) \frac{\partial p(\theta, \phi)}{\partial \theta}^{2} \cos (\phi)^{2}+\frac{\partial p(\theta, \phi)}{\dot{\partial} \theta}{\frac{\partial^{2}}{\partial \theta}(\theta, \omega)}_{\partial \phi^{2}} \cos (\phi)^{2}\right) / \cos (\phi)^{3}
\end{aligned}
$$

All these quantities are sufficient for solving the different eduations. 


\section{References}

[ALSi] N. Ayache and I: Lustman. Fast and Reliable Passive Trinorular Stercovision. In First Intromational Confrerence on Computer l'ision. lune 198.

[BBSI] H. Baker and T.O. Binford. Depth from Edge and Intensity Based Stereo. In Prorecr-

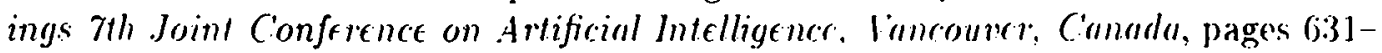
636, Augusi 1981 .

[BC90] Andrew Blake and Roberto Cipolla. Robust Estimation of Surface c 'urvature from Deformation of Apparent Contours. In First Europerun C'onferener on Compuler Vision. 1990.

[BPYA85] Michael Brady, Jean Ponce, Alan Yuille, and Harno Asada. Describing Surfaces. In Hideo Hanafusa and Hirochika Inone, editors. The Second International Symposium of Rolotics Research, pages 5-16, 1985.

[Carib] M. P. Do Carmo. Differential Cieometry of Curbes and Surfures. Prentice-Hall. Inc.. Englewood (lliffs, New Jersey, 1976 .

[Dersi] R. Deriche. Using Canny's Criteria to Derive an Optimal Edge Detector Recursively: Implemented. In The International Journal of Computer Vision, pages 15-20. April 1987.

[Fan90] Olivier D. Fangeras. On the motion of 3D curves and its relationship to optical flow. In First European Confercuce on C'ompuler Vision. 1990.

[Fl.B90] Olivier D. Pangeras. Elizabeth Lebras-Mehlman, and Jean-Danicl Boissonnat. Representing Sterco data with the Delaunay Triangulation. Artificiol Intelligence Journal. H(1-2). July 1990. also INRIA Tech. Report iss.

[F' T6] O.D. Faugeras and G. Toscani. The Calibration Problem for Stereo. In Procedings of C'IPR S6.1hirmi Bearh.Floridu, pages 15-20, 1986).

[Gir8.3] W.E.L. Grimson. An Implementation of a Computational Theory of Surface Interpolation. Computer Iision, Cirrophirs. and Image Processing, 22:39-69. 1983.

[Gris.5] W.E.L. Cirmson. Computational experiments with a feature based sterco algorithm. IEEE Transartions on Pattern Alualysis and Machine Mntclligence, $\overline{7}$ No $1: 1 \bar{i}-3-1$. 1985.

[Cills6] Peler (iiblin and R. Weiss. Reconstruction of Surfaces from Profiles. In First In/ernutional Conference on C'omputer lision. december 1986.

[Hor86] Berthold Klaus Paul Horn. Robot lision. MIT Press. 1986.

[Lioest] Jan Koenderink. What Does the Occluding Contour Tell Its About Solid Shape? Perceplion. 13:321-330, 1984.

[LI38S] Hong Seh Lim and Thomas O. Binford. Curved Surface Reconstruction Using Stereo Correpondance. In Imagc Cindf rstunding Workshop. pages 809-819, 1988.

[Mars2] D. Marr. Tision. W.H. Freman and ('o. 1982. 


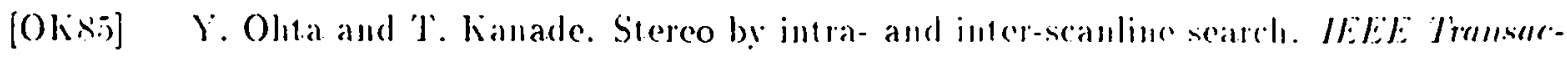

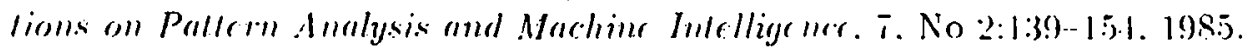

[RSEN] Basri Ronen and Lllman Shimon. The Alignement of Objects will Smonth Surfaces. In Secand International Conferrnee on Compuler lision, decomber 1988.

['Tsas(i] Roger Y. Tsai. An Efficient and Accurate Camera Cadibration l'eclunique for 3D Ma-

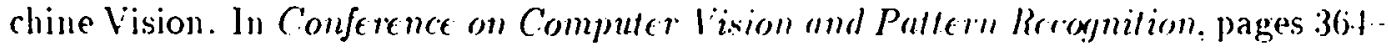
$374,1986$.

[Vaj90] Régis Vaillant. Les contours d'orcultation. Ph]) thesis. liniversité de Paris-Sud Contre d'Orsay, 1990. to appear.

[VDF\&9] Régis Vaillant, Rachid Deriche, and Olivier Fangeras. 3D Vision on the Parallel Nachine CAPITAN. In International Workshop on Industrial Application of Machine Intclligener and l'ision, april 1989.

[YTs9] Régis Vaillant and Olivier Fangeras. Trinocular Stcrcorision using lïgural Contimuiy:

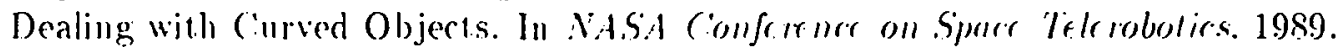


ISSN $0249-6399$ 